\title{
The 6-GHz multibeam maser survey - II. Statistical analysis and Galactic distribution of 6668-MHz methanol masers
}

\author{
J. A. Green, ${ }^{1,2 \star ~ S . ~ L . ~ B r e e n, ~}{ }^{1,3}$ G. A. Fuller, ${ }^{4,5}$ N. M. McClure-Griffiths, ${ }^{6}$ \\ S. P. Ellingsen, ${ }^{7}$ M. A. Voronkov, ${ }^{1,7,8}$ A. Avison, ${ }^{4,5}$ K. Brooks, ${ }^{1,9}$ M. G. Burton, ${ }^{10,11}$ \\ A. Chrysostomou, ${ }^{2,12}$ J. Cox, ${ }^{13}$ P. J. Diamond, ${ }^{2}$ M. D. Gray, ${ }^{4}$ M. G. Hoare,${ }^{14}$ \\ M. R. W. Masheder, ${ }^{15}$ M. Pestalozzi, ${ }^{16}$ C. Phillips, ${ }^{1}$ L. J. Quinn,,${ }^{4,17}$ \\ A. M. S. Richards, ${ }^{4,5}$ M. A. Thompson, ${ }^{12}$ A. J. Walsh, ${ }^{18}$ D. Ward-Thompson, ${ }^{13,19}$ \\ D. Wong-McSweeney ${ }^{4}$ and J. A. Yates ${ }^{20}$
}

Affiliations are listed at the end of the paper

Accepted 2017 April 7. Received 2017 April 7; in original form 2016 November 8

\begin{abstract}
The Methanol Multibeam survey has produced the largest and most complete census of methanol and excited-state hydroxyl masers in the Galaxy to date. Observing the entire Galactic plane visible from the Southern hemisphere for 6668-MHz methanol and 6030/6035MHz hydroxyl masers, to an rms sensitivity of $0.015 \mathrm{Jy} \mathrm{km} \mathrm{s}^{-1}$, the survey has detected a total of 972 methanol maser sources, implying a total Galactic population of $\sim 1290$ sources with flux densities above the survey $3 \sigma$ peak flux density limit of $0.51 \mathrm{Jy}$. We present here the statistical properties of the methanol detections of the survey, including distributions in flux density, variability and range of source velocities. The data suggest that the weaker masers exhibit greater variability. We also present an analysis of the Galactic distribution of $6668-\mathrm{MHz}$ methanol masers. For the Galactic distribution, we present kinematic distance resolutions to an additional 202 sources to those published previously, and collate these with previous allocations, as well as exploring a recent Bayesian distance approach based on maser parallaxes to separately determine distances. We examine Galactic structure and determine the luminosity function of the Galactic population of methanol masers. We find that more luminous masers have an evenly distributed wide range of velocity widths compared with less luminous masers being dominated by narrow velocity ranges, with the implication that this may be tied to the evolution of the host protostar(s). We also see an indication that brighter sources are seen towards the arm origins.
\end{abstract}

Key words: masers - surveys - stars: formation - stars: massive-Galaxy: structure.

\section{INTRODUCTION}

The completion of the Methanol Multibeam (MMB) survey with the Parkes Radio Telescope allows for the statistical analysis of the largest and most complete catalogue of 6668-MHz methanol masers to date. The survey techniques are described in Green et al. (2009a), the methanol catalogues including high-resolution positions are published in a separate series (Caswell et al. 2010; Green et al. 2010; Caswell et al. 2011; Green et al. 2012; Breen et al. 2015) and the associated excited-state hydroxyl detections are also pub-

^E-mail: james.green@csiro.au lished (Avison et al. 2016). Prior to the MMB, observations of the $6668-\mathrm{MHz}$ methanol maser had been relatively extensive, but limited to inhomogeneous targeted surveys, biased by their target selections, and untargeted surveys across limited portions of the Galaxy, with varying sensitivities. The results of these previous surveys were compiled by Malyshev \& Sobolev (2003), Xu, Zheng \& Jiang (2003) and Pestalozzi, Minier \& Booth (2005), amounting to $\sim 550$ sources. Since these compilations, a number of other surveys have been conducted, including Pandian, Goldsmith \& Deshpande (2007), Ellingsen (2007), Xu et al. (2008), Cyganowski et al. (2009) and Olmi et al. (2014), adding approximately 50 sources to those known. Statistical studies have been made of the existing samples by Xu et al. (2003), van der Walt (2005) and Pestalozzi 
et al. (2007), and include predicting a Galactic population of the order of 1200 sources. The MMB detected 972 sources across longitudes $186^{\circ}$ to $60^{\circ}$ (Caswell et al. 2010; Green et al. 2010; Caswell et al. 2011; Green et al. 2012; Breen et al. 2015), which, combined with the known sources between longitudes $60^{\circ}$ and $186^{\circ}$, gives a total known Galactic population of 1032.

In addition to the well-documented application of methanol masers in understanding the formation of high-mass $\left(\geq 8 \mathrm{M}_{\odot}\right)$ stars, the intrinsic relationship of $6668-\mathrm{MHz}$ methanol masers with high-mass star formation (HMSF) regions (Minier et al. 2003; $\mathrm{Xu}$ et al. 2008; Breen et al. 2013), and their dust content (e.g. Urquhart et al. 2015), allows for the distribution of these regions to be determined directly from the distribution of masers. This can be examined with respect to the unambiguous longitude-velocity space, as has been done for the masers towards the inner Galaxy (Green et al. 2009b, 2011) or the top-down Galactic viewpoint, the latter requiring either the adoption of a kinematic model (e.g. Green \& McClure-Griffiths 2011) or parallax measurements, such as those conducted as part of the BeSSel project (Reid et al. 2014, references therein).

This paper is divided into four sections: a brief recount of the survey together with updates; distance determinations through the method of Hi self-absorption (HiSA), adding to those of Green \& McClure-Griffiths (2011), together with distance determinations through the recent Reid et al. (2016) approach; an analysis of the global properties of the masers; and finally an analysis of their distribution in the Galaxy (i.e. the distance-dependent quantities).

\section{THE MMB SURVEY}

As described in Green et al. (2009a), the MMB survey, using the Parkes Radio Telescope, obtained a one $\sigma$ peak flux density sensitivity of $0.17 \mathrm{Jy}$ (at a spectral channel width of $0.09 \mathrm{~km} \mathrm{~s}^{-1}$ and an effective velocity resolution of $0.11 \mathrm{~km} \mathrm{~s}^{-1}$ ) across the Galactic longitude region $186^{\circ}$, through the Galactic Centre, to $60^{\circ}$, with a latitude extent of $\pm 2^{\circ}$. Sources were re-observed with targeted observations to obtain accurate spectra (so-called 'MX observations), and all new sources were followed up with observations with the Australia Telescope Compact Array (ATCA) or the MERLIN array, to obtain high-resolution positions. The survey detected 972 sources, of which 360 sources were new discoveries. The detection rate of the MMB Parkes survey was 1.03 sources $\mathrm{deg}^{-2}$ across the entire survey region and 1.91 sources $\mathrm{deg}^{-2}$ for the symmetric coverage of the inner Galaxy $\left( \pm 60^{\circ}\right)$. Previous surveys (non-targeted) have yielded detection rates between 0.22 sources $\mathrm{deg}^{-2}$ (Pestalozzi et al. 2005) and greater than 10 sources deg ${ }^{-2}$ (Caswell 1996a,b; Ellingsen et al. 1996). These factors were greatly influenced by the survey regions, in terms of both the latitude coverage (with approximately \pm 0.5 yielding higher rates than broader latitude surveys, due to the tight distribution of masers to the plane) and the longitude coverage (the lower end observed towards the outer Galaxy, the upper towards the Galactic Centre).

\subsection{Update to catalogue}

Here, we note a minor update to a source published in the MMB catalogues: The source $14.457-0.143$ requires a correction to the declination listed in Green et al. (2010), i.e. it should be listed as $-16^{\circ} 26^{\prime} 57^{\prime \prime} .5$.

\section{DISTANCES TO 6668-MHZ METHANOL MASERS}

Ideally, distances to maser sources are determined directly through astrometric observations of their parallaxes with very long baseline interferometry (Brunthaler et al. 2011). However, the data do not yet exist for the vast majority of the MMB catalogue: the most recent compilation, Reid et al. (2014), providing distances to $\sim 100$ sources north of declination $-38^{\circ} \quad(\sim 30$ per cent with negative declinations). The only methanol maser sources with astrometric parallax distances south of this declination are those in Krishnan et al. $(2015,2017)$.

As a substitute, kinematic models are used to estimate the distance. Kinematic distances have two main limitations: First, there is the ability of the kinematic model to accurately portray the Galactic rotation at any given location within the Galaxy; secondly, for sources within the solar circle, where velocities correspond to two distances, there is the non-trivial issue of resolving the ambiguity between a near and a far distance. Nevertheless, kinematic distances provide our best current estimate, and in this section, we explore two kinematic methods to determine distances to sources: The first resolves ambiguities through examining $\mathrm{H}_{\mathrm{I}}$ profiles, and the second resolves ambiguities through associating sources with spiral arms with astrometric parallax measurements. We discuss a comparison of the results of both techniques, and then utilize one method for distance-dependent population properties (presented in Section 5). For comparison, the results of the other method are shown in the online appendices.

\subsection{Kinematic distances and H ISA}

Distances to a large portion of the sources in the MMB catalogue (the first three catalogues plus previously known sources) have already been determined through the assumption of a flat rotation curve (Reid et al. 2009; McMillan \& Binney 2010) and the use of the technique of HiSA (Heeschen 1955; Burton, Liszt \& Baker 1978; Liszt, Burton \& Bania 1981) to resolve the ambiguities. This technique utilizes the association of the cold neutral medium, traced by cold $\mathrm{HI}_{\mathrm{I}}$, with molecular clouds, to determine if a cloud is in the foreground or not, relative to background emission. Distances to 442 sources were presented thus in Green \& McClure-Griffiths (2011). In this section, we resolve distances for a further 202 sources, through assessing the sources published since that study (new MMB detections in catalogues IV and V; Green et al. 2012; Breen et al. 2015, respectively).

Although kinematic distance estimators based on implications from (predominantly first and second Galactic quadrant) parallax measurements now exist (Reid et al. 2016, described in the next Section), for consistency, here we follow the same method as Green \& McClure-Griffiths (2011), taking the difference of H I spectra taken towards the source and an averaged 'off' position, together with the gradient of the spectra (the rate of change of $\mathrm{H}$ I emission with kinematic velocity) and a map.

As in Green \& McClure-Griffiths (2011), the HI 'off' spectra were the averages of data from four 3-arcmin offset positions, placed north-east, north-west, south-east and south-west of the source (in Galactic coordinates). The offset scale was computed on the basis that the maser sites are compact, typically existing in clouds with diameters of a few parsec, as other tracers of star-forming clumps and cores, such as the ATLASGAL and BGPS surveys, indicate median sizes of approximately 1 pc or less (Urquhart et al. 2014b; Svoboda et al. 2016). With typical distances greater than $2 \mathrm{kpc}$, this 
physical size equates to an approximate maximum angular size of 3 arcmin. We have used the H I data of the VLA (Very Large Array) Galactic Plane Survey (VGPS; Stil et al. 2006) and the Southern Galactic Plane Survey (SGPS; McClure-Griffiths et al. 2005), to extract the spectra at the position and velocity of the masers. We also define the same H ISA classifications as Green \& McClure-Griffiths (2011), where the 'mid-velocity' in the following criteria is the velocity at the mid-point between the lowest and highest velocity maser features assigned to a site:

(i) 'Class A' near distance assignments as ones that demonstrate an absorption dip of $\delta T>10 \mathrm{~K}$ within $10 \mathrm{~km} \mathrm{~s}^{-1}$ of the maser mid-velocity against a background $\mathrm{H}$ I emission $\geq 40 \mathrm{~K}$.

(ii) 'Class A' far distance assignments as ones that show no absorption dip with $\delta T>10 \mathrm{~K}$ within $10 \mathrm{~km} \mathrm{~s}^{-1}$ of the maser midvelocity, but demonstrate $\mathrm{H}_{\mathrm{I}}$ emission $\geq 40 \mathrm{~K}$.

(iii) 'Class B' near distance assignments as ones that have an absorption dip of $\delta T>10 \mathrm{~K}$ within $10 \mathrm{~km} \mathrm{~s}^{-1}$ of the maser mid-velocity or slightly beyond the velocity margin (within $\pm 15-$ $20 \mathrm{~km} \mathrm{~s}^{-1}$ ) and for which the width of the absorption dip may exceed $5 \mathrm{~km} \mathrm{~s}^{-1}$ (and thus the negative-positive, 's-'shaped, flip in the profile in the gradient spectrum is less prominent).

(iv) 'Class B' far distance assignments as ones that do not appear to show an absorption dip with $\delta T>10 \mathrm{~K}$ within $10 \mathrm{~km} \mathrm{~s}^{-1}$ of the maser mid-velocity, but may have weaker background $\mathrm{H}$ I emission $(<40 \mathrm{~K})$ or a small absorption $\operatorname{dip}(\delta T<10 \mathrm{~K})$ near the boundary of the $10 \mathrm{~km} \mathrm{~s}^{-1}$ margin. These allocations may also exhibit potentially confusing fluctuations in the background emission.

We reiterate here the reliability statistics that were found for this method with the SGPS and VGPS data sets by Green \& McClureGriffiths (2011), namely that for Class 'A', there is $>80$ per cent agreement with literature allocations, and for Class ' $\mathrm{B}$ ', there is $>70$ per cent agreement with literature allocations. The distances together with the Hi spectra and maps are presented in the online appendices in full (Fig. A1 and Table A1, and examples are provided in Fig. 1 and Table 1). Furthermore, we also present in the appendices (Table A2) distances that we have updated following the study of Green \& McClure-Griffiths (2011). These are principally updates for astrometric measurements that have been made in the time since that study, but also include four corrections. In total, combined with literature allocations, we obtain distances for 778 of the MMB sources.

\subsection{Parallax-based distance estimator}

In addition to the distance estimates described in the previous section, we also utilize the recent parallax-based approach presented by Reid et al. (2016), which assigns sources to spiral arms based on a Bayesian technique. Both methods have their advantages and limitations: The former is limited to those sources that exhibit $\mathrm{H}$ I spectra for which we can determine allocations, but can be utilized across the Galactic plane without bias; the latter is currently biased by a predominantly Northern hemisphere set of parallax measurements, and so its most relevant results are restricted to that hemisphere, but it can none the less be applied to the full MMB sample (technically all 972 sources, rather than the 778 of the previous section; however, for comparison, we restrict the sources to the same sample as found through the HiSA method).

For the implementation of the parallax-based approach, we follow the process described in Reid et al. (2016), utilizing the
FORTRAN program, ${ }^{1}$ and have adopted the default assumption of the spiral arm association weighting to be uniformly 0.5 , i.e. no preferential association. Briefly, this process consists of assigning a maser to a spiral arm, where the spiral arm is a continuous curve in longitude, latitude and velocity, anchored to astrometric parallax measurements. This uses a Bayesian approach to combine the probabilities (of arm association, kinematic distance association and parallax measurement association) to form a distance probability density function. The resultant distances (and their probabilities) are presented in full in the online appendices (Table B1), and an example is provided in Table 2.

\subsection{Comparison and adoption of distances}

For the 778 sources for which a distance could be determined through both methods, we found the following: 38 per cent of the distances were within the calculated error margins of one another; 49 per cent were within $1 \mathrm{kpc}$ (typical spiral arm width) of each other; and the median difference between the distances determined through the two methods was $1.1 \mathrm{kpc}$. The majority of the discrepant distances were differences in the ambiguity solutions, where one method had adopted a near distance and the other adopted a far distance. The largest distance uncertainties are found on the largest heliocentric distances, and are typically for sources for which we would exclude their distances for analysis anyway, for example, with velocities associated with the Galactic Centre. The H ISA technique does not inherently incorporate latitude implications (high latitudes most likely corresponding to near distances), and the Reid et al. (2016) approach does not currently incorporate Southern hemisphere Galactic structures, and the Galactic Centre, fully. The latter is due to the limited data in the south (four maser sources currently; Krishnan et al. 2015, 2017), and the difficulties in obtaining astrometric measurements towards the Galactic Centre. As an aside, both techniques could benefit from incorporating knowledge of the angular scale of the distribution of associated Class I methanol maser spots, where there is an indication that a tighter spot distribution corresponds to a further kinematic distance (Voronkov et al. 2014); however, this could not be included uniformly for the current sample.

Unfortunately, the current lack of Southern hemisphere maser parallaxes makes specific (and quantitative) comparative analysis of the reliability of either method for the south limited. As such, in this paper, we choose to adopt the HiSA-based distances for subsequent analysis, on qualitative grounds: We adopt the HiSA distances on the basis that the HISA method takes into account Southern hemisphere measurements, whilst the Reid et al. (2016) methods strength lies in the structures identified and measured in the Northern hemisphere; the MMB catalogue is also predominantly in the Southern hemisphere Galactic plane. Utilizing H ISA distances also ensures continuity with the previous MMB allocations (Green \& McClure-Griffiths 2011).

Although for subsequent analysis we use only the HiSA distances, for comparison, we do, however, provide the equivalent analysis plots from the Reid et al. (2016) technique for all distancedependent quantities in the online appendices (Tables B1- B3).

\footnotetext{
${ }^{1}$ Available at http://bessel.vlbi-astrometry.org
} 

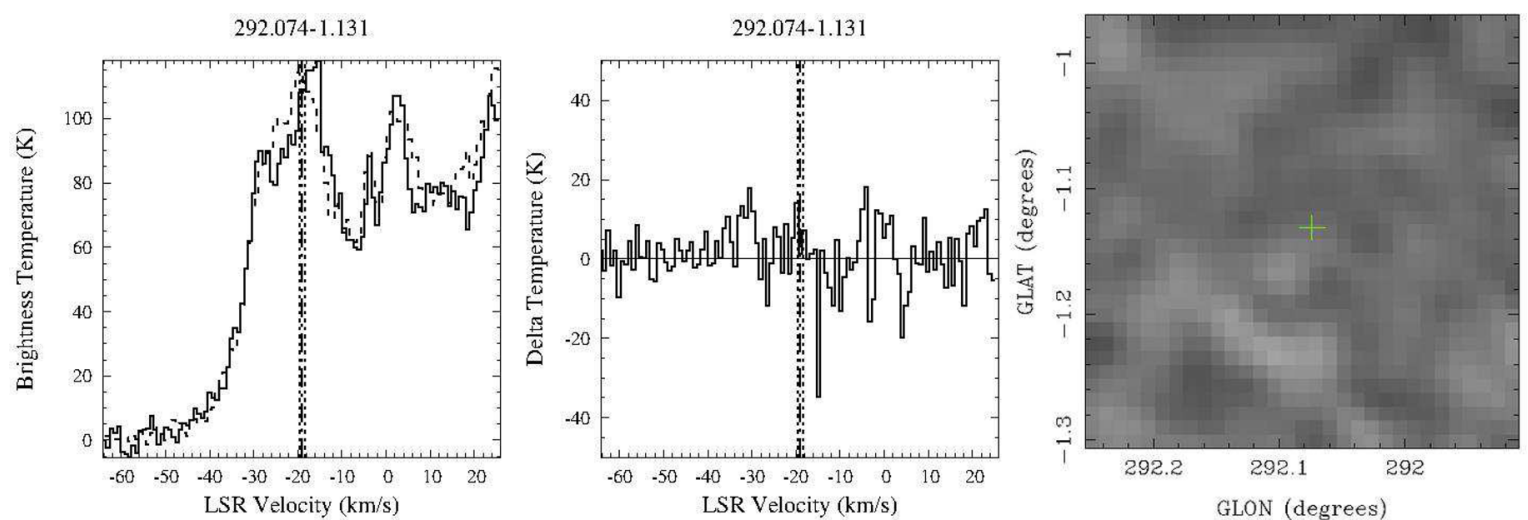

299.772-0.005
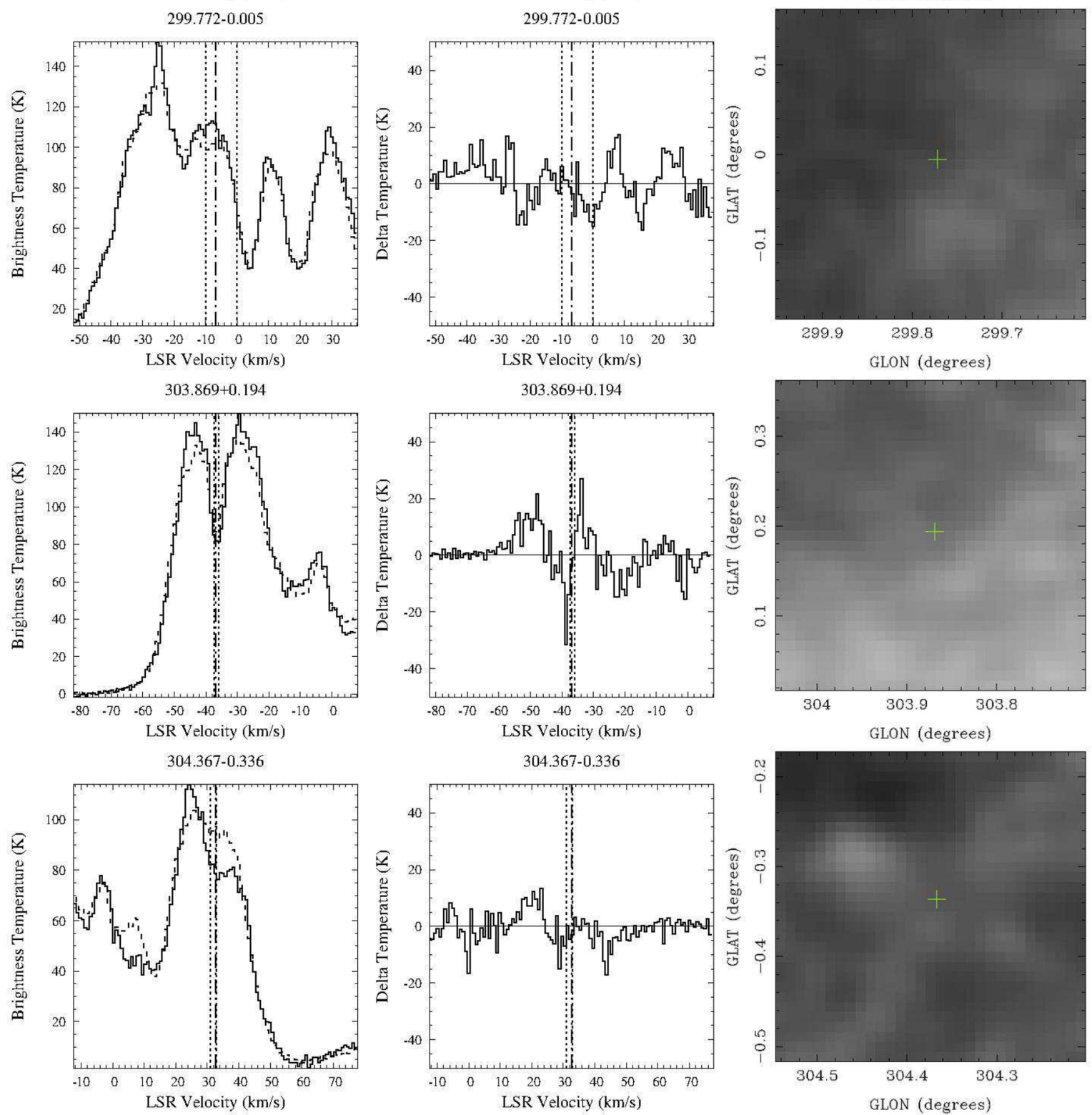

Figure 1. From the left- to right-hand side: $\mathrm{H}_{\mathrm{I}}$ spectrum on-source (solid) and off-source (dashed); gradient of the velocity of the $\mathrm{H}_{\mathrm{I}}$ spectrum (temperature change per channel); and H I map (grey scale) centred at the source position. The grey scale of the map is a linear scale from 0 (black) to $150 \mathrm{~K}$ (white). The broken vertical lines indicate the range of velocity over which maser emission can be detected, and the solid line indicates the velocity of the peak of the maser emission. Full figure is provided in the online appendices. 
Table 1. Example table of distance assignments of 6668-MHz methanol masers, not discussed in Green \& McClure-Griffiths (2011). All the sources are referenced as ${ }^{1}$ Green et al. (2012), ${ }^{2}$ Caswell et al. (2010), ${ }^{3}$ Breen et al. (2015), and references therein. H ISA class designations are (Green \& McClureGriffiths 2011) as follows: a - best H ISA distance assignments meeting criteria discussed in text; $\mathrm{b}-\mathrm{H}$ ISA distance assignments not meeting all the criteria; $\mathrm{x}$ - sources for which we were unable to determine HiSA; c - direct association with a continuum source; o - velocity or longitude implies outer Galaxy source; and $\mathrm{t}$ - velocity results in tangent point distance. All kinematic distances are calculated using a flat rotation curve with $\theta$ of $246 \mathrm{~km} \mathrm{~s}{ }^{-1}$ and $R_{\odot}$ of $8.4 \mathrm{kpc}$. The mid-point LSR velocity of the maser is used after it has been corrected for the best estimates of the solar motion: $U_{\odot}=11.1, V_{\odot}=12.2$ and $W_{\odot}=7.25 \mathrm{~km} \mathrm{~s}^{-1}$ (Reid et al. 2009; McMillan \& Binney 2010; Schönrich, Binney \& Dehnen 2010). Where distance resolutions have been made previously in the literature, they are listed (last column) as follows: ${ }^{\mathrm{CAS}}$ Caswell et al. (1975); ${ }^{\mathrm{KUC}} \mathrm{Kuchar} \&$ Bania (1994); ${ }^{\mathrm{KAW}} \mathrm{Kawamura}$ et al. (1998); ${ }^{\mathrm{ARA}} \mathrm{Araya}$ et al. (2002); ${ }^{K O L}$ Kolpak et al. (2003); ${ }^{\text {FIS }}$ Fish et al. (2003); ${ }^{\text {MEN }}$ Menten et al. (2007); ${ }^{\text {REIl }}$ Reid et al. (2009); ${ }^{\text {REI2 }}$ Reid et al. (2009); ${ }^{\text {ZHAl }}$ Zhang et al. (2009);

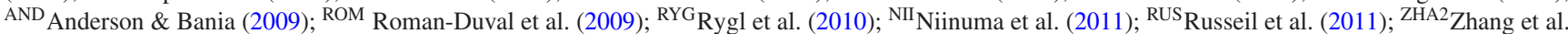
(2013); ${ }^{\text {SAT }}$ Sato et al. (2014); and ${ }^{\mathrm{WU}} \mathrm{Wu}$ et al. (2014). We have ignored resolutions from the literature labelled as 'tangent point'. References prefixed with an 'A' are astrometric distances. For sources with astrometric parallax distances, only the parallax measurement is referenced. The kinematic distance errors are calculated from the uncertainties in the velocities and include a systematic error of $\pm 7 \mathrm{~km} \mathrm{~s}^{-1}$ as detailed in Reid et al. (2009). For the purposes of this work, the 3-kpc arm sources are arbitrarily positioned on a circle of radius $3.4 \mathrm{kpc}$ from the Galactic Centre. $V_{\mathrm{p}}$ is the velocity of the peak emission feature and $V_{\mathrm{m}}$ is the mid-point of the velocity range over which emission is seen. Full table is provided in the online appendices; see Table A1.

\begin{tabular}{|c|c|c|c|c|c|c|c|c|c|}
\hline \multicolumn{3}{|c|}{ 6668-MHz methanol maser } & \multicolumn{2}{|c|}{ HiSA } & \multirow{2}{*}{$\begin{array}{c}\text { Source } \\
\text { status }\end{array}$} & \multirow{2}{*}{$\begin{array}{l}\text { Dist. } \\
(\mathrm{kpc})\end{array}$} & \multicolumn{2}{|c|}{ Error } & \multirow{2}{*}{$\begin{array}{c}\text { Previous } \\
\text { allocation }\end{array}$} \\
\hline$\left({ }^{\circ}\right)$ & $\left(\begin{array}{c}\mathrm{p}^{\mathrm{p}} \\
\left(\mathrm{kms}^{-1}\right)\end{array}\right.$ & $\left(\mathrm{kms}^{-1}\right)$ & & & & & $(\mathrm{kpc})$ & $(\mathrm{kpc})$ & \\
\hline $188.794+1.031^{1}$ & -5.5 & -5.0 & - & $\mathrm{o}$ & Outer Galaxy, literature & 2.0 & 0.1 & -0.1 & $2^{\mathrm{NII}}$ \\
\hline $188.946+0.886^{1}$ & 10.8 & 3.5 & - & o & Outer Galaxy, literature & 2.1 & 0.0 & -0.0 & $\mathrm{~A}^{\mathrm{REI} 2}$ \\
\hline $189.030+0.783^{1}$ & 8.9 & 9.2 & - & o & Outer Galaxy, literature & 2.0 & 1.0 & -1.0 & $2^{\mathrm{KAW}}$ \\
\hline $189.471-1.216^{1}$ & 18.8 & 19.0 & - & o & Outer Galaxy, literature & 2.0 & 1.0 & -1.0 & $2^{\mathrm{KAW}}$ \\
\hline $189.778+0.345^{1}$ & 5.7 & 4.0 & - & o & Outer Galaxy, literature & 2.0 & 1.0 & -1.0 & $2^{\mathrm{KAW}}$ \\
\hline $192.600-0.048^{1}$ & 4.6 & 3.5 & - & o & Outer Galaxy, literature & 1.6 & 0.1 & -0.1 & $\mathrm{~A}^{\mathrm{RYG}}$ \\
\hline $196.454-1.677^{1}$ & 15.2 & 14.8 & - & o & Outer Galaxy, literature & 5.3 & 0.2 & -0.2 & $\mathrm{~A}^{\mathrm{HON}}$ \\
\hline $206.542-16.355^{1}$ & 12.3 & 12.3 & - & o & Outer Galaxy, literature & 0.4 & 0.0 & -0.0 & $\mathrm{~A}^{\mathrm{MEN}}$ \\
\hline $208.996-19.386^{1}$ & 7.3 & 7.5 & - & o & Outer Galaxy, literature & 0.4 & 0.0 & -0.0 & $\mathrm{~A}^{\mathrm{MEN}}$ \\
\hline $209.016-19.398^{1}$ & -1.5 & -1.0 & - & o & Outer Galaxy, literature & 0.4 & 0.0 & -0.0 & $\mathrm{~A}^{\mathrm{MEN}}$ \\
\hline $212.067-0.750^{1}$ & 44.4 & 45.8 & - & o & Outer Galaxy & 5.2 & 1.3 & -1.1 & \\
\hline $213.705-12.597^{1}$ & 10.7 & 11.0 & - & o & Outer Galaxy, literature & 0.4 & 0.0 & -0.0 & $\mathrm{~A}^{\mathrm{MEN}}$ \\
\hline $232.620+0.996^{1}$ & 22.9 & 21.5 & - & o & Outer Galaxy, literature & 1.7 & 0.1 & -0.1 & $\mathrm{~A}^{\mathrm{REI} 2}$ \\
\hline $254.880+0.451^{1}$ & 30.1 & 30.3 & - & o & Outer Galaxy & 3.2 & 0.6 & -0.6 & \\
\hline $259.939-0.041^{1}$ & -1.0 & -1.0 & - & o & Outer Galaxy & 0 & 1 & -0 & \\
\hline $263.250+0.514^{1}$ & 12.3 & 14.3 & - & o & Outer Galaxy & 2.5 & 0.7 & -0.8 & \\
\hline $264.140+2.018^{1}$ & 8.1 & 8.3 & - & o & Outer Galaxy & 1.9 & 0.8 & -1.0 & \\
\hline $264.289+1.469^{1}$ & 8.7 & 7.8 & - & o & Outer Galaxy & 1.9 & 0.8 & -1.0 & \\
\hline $269.153-1.128^{1}$ & 16.0 & 12.0 & - & o & Outer Galaxy & 3.0 & 0.7 & -0.9 & \\
\hline $269.456-1.467^{1}$ & 56.1 & 55.0 & - & o & Outer Galaxy & 7.0 & 0.6 & -0.6 & \\
\hline $269.658-1.270^{1}$ & 16.2 & 15.3 & - & o & Outer Galaxy & 3.4 & 0.7 & -0.8 & \\
\hline $281.710-1.104^{1}$ & 0.9 & 1.8 & - & o & Outer Galaxy & 4.1 & 0.8 & -0.8 & \\
\hline $284.694-0.361^{1}$ & 13.3 & 13.0 & - & o & Outer Galaxy & 6.0 & 0.6 & -0.7 & \\
\hline $286.383-1.834^{1}$ & 9.6 & 9.3 & - & o & Outer Galaxy & 6.1 & 0.6 & -0.7 & \\
\hline $290.374+1.661^{1}$ & -24.2 & -25.0 & & & & & & & \\
\hline $290.411-2.915^{1}$ & -16.0 & -16.0 & & & & & & & \\
\hline $291.642-0.546^{1}$ & 12.1 & 12.0 & - & $\mathrm{o}$ & Outer Galaxy & 7.6 & 0.6 & -0.6 & \\
\hline $291.879-0.810^{1}$ & 33.5 & 32.3 & - & o & Outer Galaxy & 9.3 & 0.6 & -0.6 & \\
\hline $292.074-1.131^{1}$ & -19.1 & -19.0 & $\mathrm{~F}$ & $\mathrm{~b}$ & HiSA far & 4.2 & 1.1 & -1.1 & \\
\hline $292.468+0.168^{1}$ & 10.9 & 16.0 & - & o & Outer Galaxy & 8.1 & 0.6 & -0.6 & \\
\hline $293.723-1.742^{1}$ & 24.2 & 24.5 & - & o & Outer Galaxy & 9.1 & 0.6 & -0.6 & \\
\hline $294.337-1.706^{1}$ & -11.7 & -11.8 & - & $\mathrm{x}$ & Latitude near & 0 & 0 & -0 & \\
\hline $294.511-1.621^{1}$ & -11.9 & -9.0 & - & $\mathrm{x}$ & Latitude near & 0 & 6 & -0 & \\
\hline $294.977-1.734^{1}$ & -5.3 & -6.0 & - & $\mathrm{x}$ & Latitude near & 0 & 7 & -0 & \\
\hline $294.990-1.719^{1}$ & -12.3 & -12.3 & - & $\mathrm{x}$ & Latitude near & 0 & 0 & -0 & \\
\hline $297.406-0.622^{1}$ & 27.8 & 27.0 & - & o & Outer Galaxy & 10.1 & 0.6 & -0.6 & \\
\hline $298.177-0.795^{1}$ & 23.5 & 25.3 & - & o & Outer Galaxy & 10.2 & 0.6 & -0.6 & \\
\hline $298.632-0.362^{1}$ & 38.7 & 41.0 & - & o & Outer Galaxy & 11.6 & 0.6 & -0.6 & \\
\hline $298.723-0.086^{1}$ & 23.5 & 19.5 & - & o & Outer Galaxy & 9.9 & 0.6 & -0.6 & \\
\hline $299.772-0.005^{1}$ & -6.8 & -5.0 & $\mathrm{~F}$ & $\mathrm{~b}$ & H ISA far & 8.2 & 0.6 & -0.6 & \\
\hline $302.034+0.625^{1}$ & -39.1 & -44.3 & & & & & & & \\
\hline $302.455-0.741^{1}$ & 32.6 & 35.0 & - & o & Outer Galaxy & 12.0 & 0.6 & -0.6 & \\
\hline $303.507-0.721^{1}$ & 14.2 & 14.5 & - & o & Outer Galaxy & 10.6 & 0.5 & -0.5 & \\
\hline $303.846-0.363^{1}$ & 25.4 & 27.8 & - & o & Outer Galaxy & 11.7 & 0.6 & -0.6 & \\
\hline $303.869+0.194^{1}$ & -36.9 & -36.8 & $\mathrm{~N}$ & $\mathrm{~b}$ & H ISA near & 2.9 & 0.7 & -0.7 & \\
\hline
\end{tabular}


Table 2. Example table of distances derived using the technique of Reid et al. (2016). Following the technique, the last two columns are the probability of the arm allocations and the allocations themselves with the following notations: UA, unassociated; Out, outer arm; Per, Perseus arm; Loc, local arm; SgN, Sagittarius arm (near portion); SgF, Sagittarius arm (far portion); ScN, Scutum arm (near portion); ScF, Scutum arm (far portion); Nor, Norma arm; 3kN, near 3-kpc arm; $3 \mathrm{kF}$, far 3-kpc arm; AqS, Aquila spur; and LoS, local spur. $V_{\mathrm{p}}$ is the velocity of the peak emission feature. Full table is in the online appendix (Table B1).

\begin{tabular}{|c|c|c|c|c|c|}
\hline $\begin{array}{l}\text { 6668-MHz methanol maser } \\
\qquad \begin{array}{l}l b \\
\left({ }^{\circ}\right)\end{array}\end{array}$ & $\begin{array}{c}V_{\mathrm{p}} \\
\left(\mathrm{kms}^{-1}\right)\end{array}$ & $\begin{array}{l}\text { Distance } \\
(\mathrm{kpc})\end{array}$ & $\begin{array}{l}\text { Error } \\
(\mathrm{kpc})\end{array}$ & Probability & $\begin{array}{c}\text { Arm } \\
\text { allocation }\end{array}$ \\
\hline $188.794+1.031$ & -5.5 & 2.0 & 0.3 & 0.91 & Per \\
\hline $188.946+0.886$ & 10.8 & 2.1 & 0.1 & 0.95 & Per \\
\hline $189.030+0.783$ & 8.9 & 2.1 & 0.1 & 0.94 & Per \\
\hline $189.471-1.216$ & 18.8 & 2.0 & 0.1 & 0.86 & Per \\
\hline $189.778+0.345$ & 5.5 & 2.0 & 0.1 & 0.81 & Per \\
\hline $192.600-0.048$ & 4.6 & 1.6 & 0.1 & 0.73 & Per \\
\hline $196.454-1.677$ & 15.2 & 2.2 & 0.4 & 0.90 & Per \\
\hline $206.542-16.355$ & 12.3 & 0.4 & 0.1 & 1.00 & Loc \\
\hline $208.996-19.386$ & 7.3 & 0.8 & 0.0 & 1.00 & UA \\
\hline $209.016-19.398$ & -1.5 & 0.8 & 0.0 & 1.00 & UA \\
\hline $212.067-0.750$ & 43.3 & 2.8 & 0.4 & 0.84 & Per \\
\hline $213.705-12.597$ & 10.7 & 0.8 & 0.1 & 1.00 & UA \\
\hline $232.620+0.996$ & 22.9 & 1.7 & 0.1 & 0.73 & UA \\
\hline $254.880+0.451$ & 30.1 & 3.0 & 0.7 & 1.00 & UA \\
\hline $259.939-0.041$ & -1.0 & 1.1 & 0.8 & 1.00 & UA \\
\hline $263.250+0.514$ & 12.3 & 2.1 & 0.8 & 1.00 & UA \\
\hline $264.140+2.018$ & 8.1 & 1.6 & 0.8 & 1.00 & UA \\
\hline $264.289+1.469$ & 8.7 & 1.8 & 0.8 & 1.00 & UA \\
\hline $269.153-1.128$ & 16.0 & 3.0 & 0.9 & 1.00 & UA \\
\hline $269.456-1.467$ & 56.1 & 6.7 & 0.7 & 1.00 & UA \\
\hline $269.658-1.270$ & 16.2 & 3.1 & 0.9 & 1.00 & UA \\
\hline $270.255+0.835$ & 3.9 & 2.0 & 1.0 & 1.00 & UA \\
\hline $281.710-1.104$ & 0.9 & 3.0 & 1.5 & 1.00 & UA \\
\hline $284.352-0.419$ & 3.9 & 4.4 & 1.1 & 1.00 & UA \\
\hline $284.694-0.361$ & 13.3 & 5.6 & 0.8 & 1.00 & UA \\
\hline $285.337-0.002$ & 0.7 & 4.3 & 1.2 & 1.00 & UA \\
\hline $286.383-1.834$ & 9.6 & 1.2 & 0.9 & 0.58 & UA \\
\hline $287.371+0.644$ & -1.9 & 4.3 & 1.5 & 1.00 & UA \\
\hline $290.374+1.661$ & -24.2 & 2.0 & 0.8 & 1.00 & UA \\
\hline $290.411-2.915$ & -16.0 & 1.1 & 0.5 & 1.00 & UA \\
\hline $291.270-0.719$ & -31.2 & 3.2 & 1.3 & 1.00 & UA \\
\hline $291.274-0.709$ & -29.7 & 3.2 & 1.2 & 1.00 & UA \\
\hline $291.579-0.431$ & 15.2 & 7.4 & 0.7 & 1.00 & UA \\
\hline $291.582-0.435$ & 10.4 & 7.0 & 0.7 & 1.00 & UA \\
\hline $291.642-0.546$ & 12.1 & 7.2 & 0.7 & 1.00 & UA \\
\hline $291.879-0.810$ & 33.5 & 9.1 & 0.7 & 1.00 & UA \\
\hline $292.074-1.131$ & -19.1 & 2.6 & 1.1 & 1.00 & UA \\
\hline $292.468+0.168$ & 10.9 & 7.2 & 0.7 & 1.00 & UA \\
\hline $293.723-1.742$ & 24.2 & 9.1 & 0.7 & 0.74 & UA \\
\hline $293.827-0.746$ & 37.0 & 9.8 & 0.7 & 1.00 & UA \\
\hline $293.942-0.874$ & 41.1 & 10.2 & 0.7 & 1.00 & UA \\
\hline $294.337-1.706$ & -11.7 & 1.4 & 0.7 & 1.00 & UA \\
\hline $294.511-1.621$ & -11.9 & 1.4 & 0.7 & 1.00 & UA \\
\hline $294.977-1.734$ & -5.3 & 1.0 & 0.6 & 1.00 & UA \\
\hline $294.990-1.719$ & -12.3 & 1.4 & 0.6 & 1.00 & UA \\
\hline $296.893-1.305$ & 22.2 & 9.6 & 0.6 & 0.89 & UA \\
\hline $297.406-0.622$ & 27.8 & 9.9 & 0.7 & 1.00 & UA \\
\hline $298.177-0.795$ & 23.5 & 9.8 & 0.6 & 1.00 & UA \\
\hline $298.213-0.343$ & 33.3 & 10.4 & 0.7 & 1.00 & UA \\
\hline $298.262+0.739$ & -30.1 & 3.7 & 1.3 & 1.00 & UA \\
\hline $298.632-0.362$ & 37.2 & 10.9 & 0.7 & 1.00 & UA \\
\hline $298.723-0.086$ & 23.5 & 9.8 & 0.7 & 1.00 & UA \\
\hline $299.013+0.128$ & 18.3 & 9.4 & 0.7 & 1.00 & UA \\
\hline $299.772-0.005$ & -6.8 & 7.7 & 0.6 & 0.91 & UA \\
\hline
\end{tabular}




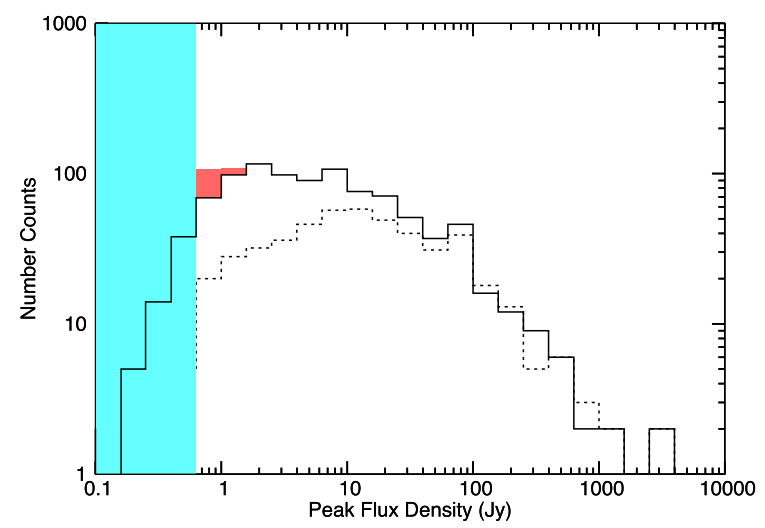

Figure 2. Peak flux density distribution for the MMB Parkes survey in logarithmic bins (in logarithmic intervals of 0.2). The dashed line represents those sources detected which were previously known, and the solid line represents the total catalogue (known and new). The blue shading represents the $3 \sigma$ sensitivity (at a velocity resolution of $0.11 \mathrm{~km} \mathrm{~s}^{-1}$ ) of the MMB. The lowest bins above the sensitivity limit include red bars showing adjustments for expected incompleteness (Green et al. 2009a).

\section{OVERALL PROPERTIES OF 6668-MHZ METHANOL MASERS}

In this section, we detail the statistics and properties of the 6668$\mathrm{MHz}$ methanol masers detected in the MMB survey, which are not dependent on calculating a source distance. We utilize the 'MX' spectral data (Green et al. 2009a) for properties such as peak flux density and peak velocities for the majority of sources; however, nine sources of the 972 did not have MX data (see individual catalogues for details), and for these, we utilize the survey spectral data as a proxy. Three sources associated with Sgr B2 do not have individual listed flux densities due to confusion, and these are also excluded from subsequent flux density analysis (and the luminosity analysis of Section 5).

\subsection{Peak flux density distribution}

The brightest methanol maser known is still the well-studied 9.621+0.196 (with an MMB-recorded flux density of $\sim 5200 \mathrm{Jy}$ ). There are 51 masers with peak flux densities above $100 \mathrm{Jy}, 330$ above $10 \mathrm{Jy}$ and 118 with peak flux densities below $1 \mathrm{Jy}$, approximately 5 per cent, 34 per cent and 12 per cent of the total population, respectively. Fig. 2 shows the distribution of peak flux density versus number counts for the population of $6668 \mathrm{MHz}$ methanol masers detected in the MMB survey. Although the detections suggest a flux turnover of around 1-2 Jy, taking into account incompleteness (Green et al. 2009a), and statistical errors, the turnover is not statistically significant. As alluded to in the technique paper (Green et al. 2009a), the potential flux turnover will be addressed with the results and analysis of the deeper 'piggyback' survey (Ellingsen et al., in preparation).

\subsection{Integrated flux density distribution}

The integrated flux densities of the MMB sources were derived and presented in the fifth catalogue paper (Breen et al. 2015), and their distribution is shown in Fig. 3. The brightest integrated flux is $\sim 5000 \mathrm{Jy} \mathrm{km} \mathrm{s}^{-1}$ for $323.740-0.263$. There are 66 sources with integrated fluxes in excess of $100 \mathrm{Jy} \mathrm{km} \mathrm{s}^{-1}$ and 203 sources with integrated fluxes less than $1 \mathrm{Jy} \mathrm{km} \mathrm{s}{ }^{-1}$. The detection threshold of

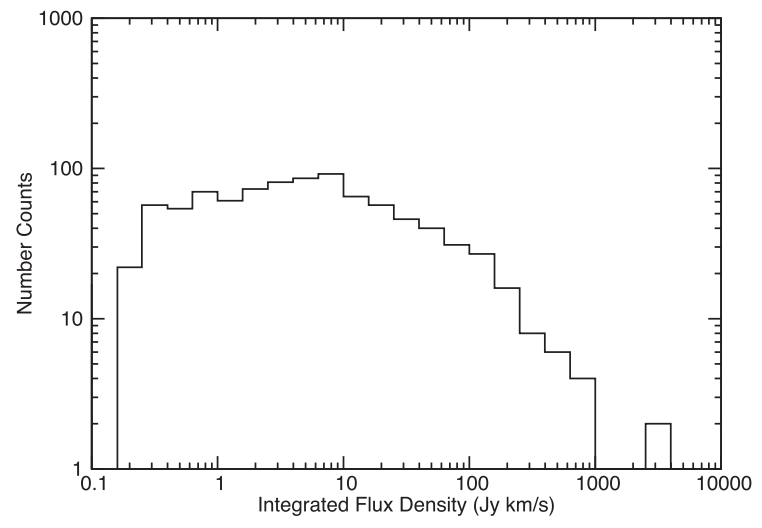

Figure 3. Integrated flux density distribution for the MMB Parkes survey in logarithmic bins (in logarithmic intervals of 0.2). Individual integrated flux densities are derived and presented in Breen et al. (2015).

the MMB of three channels with emission greater than 4.7 times the root-mean-square (rms) deviation of their respective baseline fits (Green et al. 2009a) equates to $\sim 0.15 \mathrm{Jy} \mathrm{km} \mathrm{s}^{-1}$, providing significant uncertainty to the lowest bin of Fig. 3. Above this bin, there appears to be a broken power-law distribution, with the break at $10 \mathrm{Jy} \mathrm{km} \mathrm{s}^{-1}$, and a fit below giving an index of $0.22 \pm 0.04$ and a fit above giving an index of $-0.51 \pm 0.03$.

\subsection{Distribution of source velocities}

The distribution in maser peak velocity (measured relative to the local standard of rest) with Galactic longitude for the inner $60^{\circ}$ is given in Fig. 4. The maser peak velocity is closely related to the systemic velocity of the star formation site it is tracing (e.g. Green et al. 2011), resulting in maser velocities that follow Galactic rotation, with predominantly positive velocities between $0^{\circ}$ and $+60^{\circ}$, and negative velocities between $-60^{\circ}$ and $0^{\circ}$. The distribution is therefore best analysed in relation to Galactic structure (see Section 5). Overall though, there are 118 sources (12 per cent of the MMB catalogue) with source velocities within $10 \mathrm{~km} \mathrm{~s}^{-1}$ of zero, and therefore subject to large ambiguities with respect to Galactic rotation (and thus excluded from later distance-dependent analysis), and there are 68 sources ( 7 per cent of the MMB catalogue) with velocities greater than $100 \mathrm{~km} \mathrm{~s}^{-1}$ (i.e. $|v|>100 \mathrm{~km} \mathrm{~s}^{-1}$ ). A total of 75 per cent of the low-velocity subsample are within half a degree of the Galactic plane, compared with 97 per cent of the high-velocity subsample, which is likely a reflection of the proximity of the two samples, with the former nearby and the latter towards the inner Galaxy (corroborated by the fact that all lie within $\pm 35^{\circ}$ Galactic longitude).

\subsection{Velocity widths of emission}

We define the velocity width of emission as the range (difference) in velocity between the lowest and highest velocity emission features detected and attributed to a single maser source. The widest such velocity range is $28.5 \mathrm{~km} \mathrm{~s}^{-1}$ for $305.475-0.096$, the narrowest range is $0.3 \mathrm{~km} \mathrm{~s}^{-1}$ for $37.767-0.214$ and the median range across the whole MMB sample is $6.0 \mathrm{~km} \mathrm{~s}^{-1}$ (mean is $7.2 \mathrm{~km} \mathrm{~s}^{-1}$ ). This is narrower than the result of $\mathrm{Xu}$ et al. (2003), who found with a sample of 482 masers, velocity ranges up to $71 \mathrm{~km} \mathrm{~s}^{-1}$ and a median of $8.2 \mathrm{~km} \mathrm{~s}^{-1}$. The source with a velocity reported by $\mathrm{Xu}$ et al. (2003) as $71 \mathrm{~km} \mathrm{~s}^{-1}$ wide, $31.86+0.12$, is in the MMB catalogue (listed as $30.851+0.123$ ) only $10 \mathrm{~km} \mathrm{~s}^{-1}$ wide, with higher velocity 


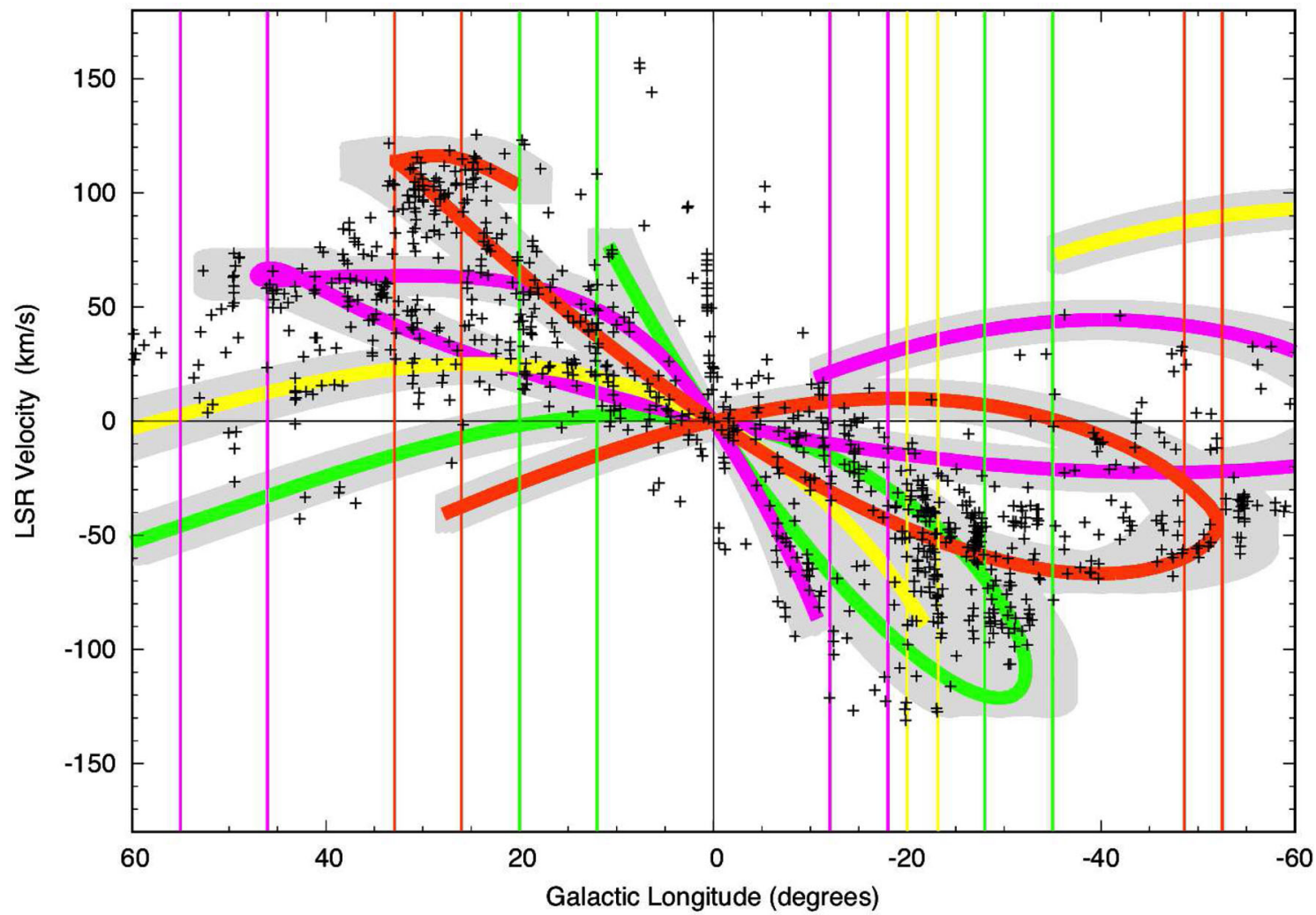

Figure 4. Longitude-velocity distribution of the $6668-\mathrm{MHz}$ methanol masers of the MMB (black crosses) for inner $60^{\circ}$ of Galactic longitude. Coloured loci represent spiral arms of the logarithmic spiral arm model of Taylor \& Cordes (1993), transferred to the longitude-velocity domain through a flat rotation curve with a circular rotation of $246 \mathrm{~km} \mathrm{~s}^{-1}$ and a Galactocentric solar distance of $8.4 \mathrm{kpc}$. Yellow loci represent the Perseus spiral arm; purple - Carina-Sagittarius; orange - Crux-Scutum; and green - Norma. The grey shading represents an error margin on the arm loci: an arm thickness of $1 \mathrm{kpc}$ and a velocity tolerance of $7 \mathrm{~km} \mathrm{~s}^{-1}$. The colour-coded pairs of vertical lines represent the arm tangents and origins as collated through a range of tracers by Vallée (2016). From the leftto right-hand side, they delineate the following: Carina-Sagittarius tangent; Crux-Scutum tangent; Norma origin; Carina-Sagittarius origin; Perseus origin; Norma tangent; and Crux-Scutum tangent.

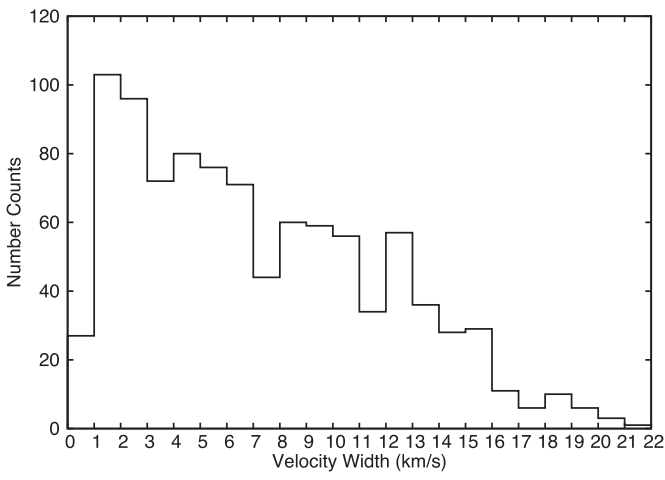

Figure 5. Distribution of velocity widths of emission for all sources of the MMB catalogue in $1 \mathrm{~km} \mathrm{~s}^{-1}$ bins. The $0-1 \mathrm{~km} \mathrm{~s}^{-1}$ bin is subject to incompleteness, and the horizontal axis has been truncated at $22 \mathrm{~km} \mathrm{~s}^{-1}$ for ease of presentation (excluding six sources with velocity widths wider than $22 \mathrm{~km} \mathrm{~s}^{-1}$ ).

features attributed to four nearby maser sources (30.818-0.057, $30.818+0.273,30.822-0.053$ and $30.898+0.161)$.

The overall distribution of velocity widths is given in Fig. 5, demonstrating a steady decline in number counts with width, meaning that we do not see clear evidence for two populations, between 010 and $10-20 \mathrm{~km} \mathrm{~s}^{-1}$, as postulated by Xu et al. (2003) and attributed, respectively, to disc and outflow-tracing populations. We

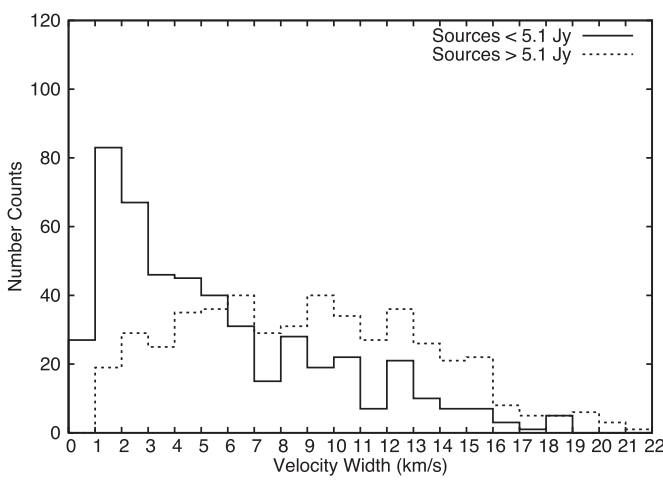

Figure 6. Distribution of velocity widths of emission for the brighter 50 per cent $(>5.1 \mathrm{Jy})$ and the fainter 50 per cent $(<5.1 \mathrm{Jy})$ of the MMB catalogue, with the horizontal axis truncated to $22 \mathrm{~km} \mathrm{~s}^{-1}$ for ease of presentation (excluding six sources with velocity widths wider than $22 \mathrm{~km} \mathrm{~s}^{-1}$ ). The fainter samples show a clear peak at $1-3 \mathrm{~km} \mathrm{~s}^{-1}$, whilst the brighter samples are more evenly spread.

find 710 sources with velocity widths between 0 and $10 \mathrm{~km} \mathrm{~s}^{-1}$ and 251 sources with velocity widths between 10 and $20 \mathrm{~km} \mathrm{~s}^{-1}$ (with only 11 sources showing emission over ranges wider than $20 \mathrm{~km} \mathrm{~s}^{-1}$ ). A drop is seen for the smallest width bin, $1 \mathrm{~km} \mathrm{~s}^{-1}$, a likely result of the incompleteness for the weakest features. The breakdown by flux density is given in Fig. 6 showing the brighter 


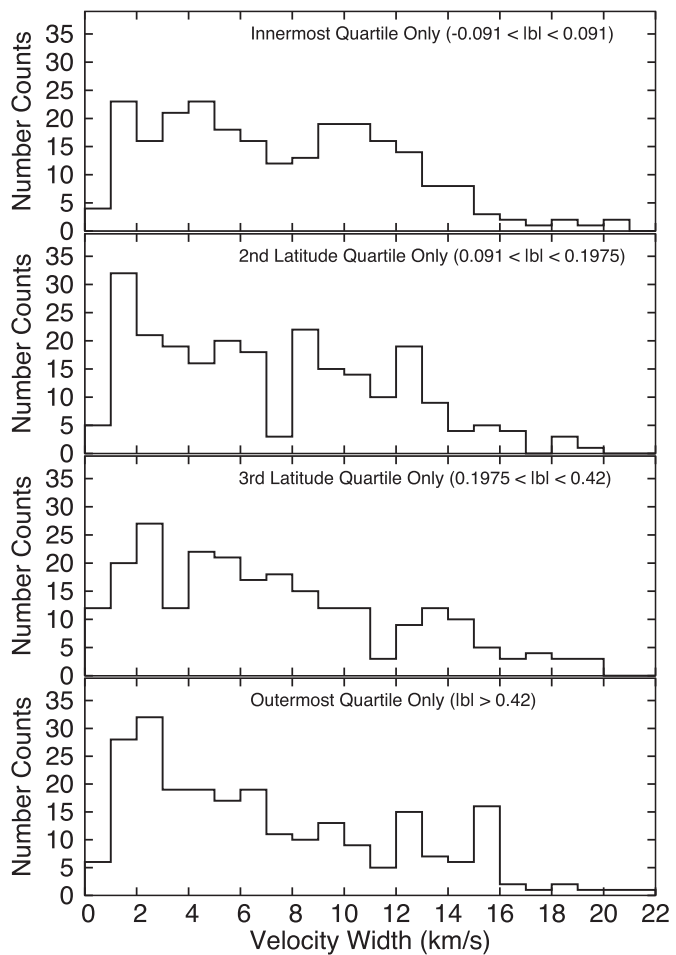

Figure 7. Distribution of velocity widths broken down in absolute Galactic latitude quartiles. Smaller latitudes exhibit a broader spread, with a less pronounced narrow-width peak.

50 per cent and weaker 50 per cent, and demonstrating an evenly distributed brighter sample but the tendency for the weaker features to have narrower emission (a peak in number counts for $1-2 \mathrm{~km} \mathrm{~s}^{-1}$ for the weakest sources). The weak peak could either be an intrinsic feature of weaker maser emission or more likely a limitation of sensitivity and will be further explored with the piggyback sample (Ellingsen et al., in preparation). The distribution with Galactic latitude is shown in Fig. 7, and shows a slight tendency for narrower emission ranges with larger latitudes. Velocity widths are further explored in Section 5 in relation to Galactic structure and derived luminosities.

\subsection{Source variability}

The majority of sources detected in the MMB survey were observed on at least two epochs, and sometimes three or four times, allowing us to assess the overall level of temporal variability in the 6668$\mathrm{MHz}$ methanol maser population. The survey observations and the follow-up MXs were typically separated by two years. Fig. 8 shows a comparison of the peak flux densities measured from the survey cube and follow-up MX observations.

The median ratio of measured MX peak flux density to survey cube peak flux density is 0.95 . Within individual portions of the catalogue, we have found this ratio to be $0.92,0.92,0.98,0.99$ and 0.89 , each time attributing the decrease in flux density in the follow-up MXs to the smaller beam (3.2 arcmin compared to the smoothed 4.4-arcmin beam of the survey cube data) and therefore the greater impact of pointing errors on the MX observations, combined with the higher noise in the survey cube data. This was compounded in the $20^{\circ}-60^{\circ}$ longitude range, where a number of followup MXs were pointed towards the survey cube position, rather than

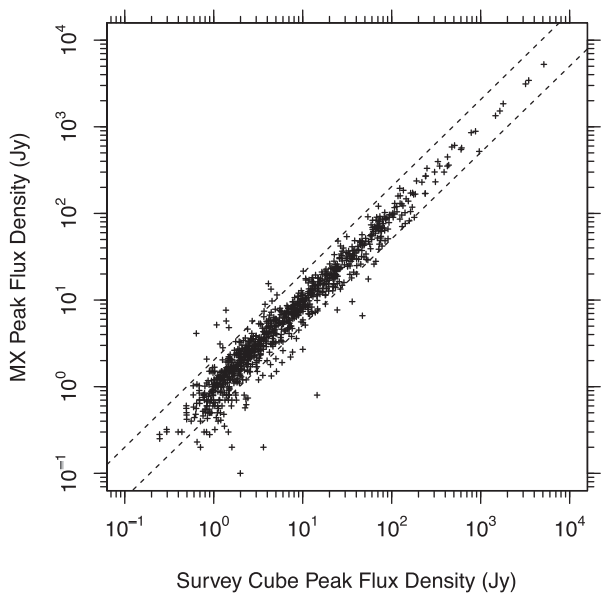

Figure 8. Peak flux density measured in the survey cube compared to the follow-up MX observations for the entire MMB longitude range. Sources that lie outside the dashed lines have varied by more than a factor of 2 .

precise positions determined from interferometric observations, as was standard practice.

Between the time the survey cube and the MX observations were made, 15 sources increased by a factor of 2 or more, and 53 decreased by a factor of 2 or more. This equates to 7 per cent of the MMB sample showing variability of a factor of 2 or more, which is consistent with the more dedicated variability study of Caswell, Vaile \& Ellingsen (1995), who found that, even in the 48 sources selected from Caswell et al. (1995) on the basis of relatively high levels of variability, flux density changes of more than a factor of 2 were not common.

In the MMB survey, there are five sources that decreased by a factor of 5 or more, the greatest change being by a factor of 20 , corresponding to 294.977-1.734, which was detected with a peak flux density of $2 \mathrm{Jy}$ in the survey cube and then fell below $0.1 \mathrm{Jy}$ in the MX observations. The other four sources exhibiting more than a factor of 5 decrease are 294.337-1.706, 14.230-0.509, $358.980+0.084$ and $45.071+0.132$. There were two sources that increased in peak flux density by more than a factor of 5 and these were $269.153-1.128$ and $264.140+2.018$, which varied by factors of 6.3 and 5.4, respectively. In total, we find that 580 sources showed a decrease in peak flux density and 356 sources showed an increase, at median rates of 24 and 28 per cent, respectively.

Comparing only the peak flux densities of two epochs will underestimate the true variability of the strongest feature measured in the survey cube in the case where a different velocity feature becomes the peak feature in the MX observation. The peak feature from the two epochs differs by more than $0.2 \mathrm{~km} \mathrm{~s}^{-1}$ in 15 per cent of sources in the entire MMB range, showing that the peak feature tends to remain consistent. This percentage is slightly higher ( 25 per cent) for the sources that have varied by more than a factor of 2 , indicating that these sources show higher levels of variability across multiple features.

Breen et al. (2012) investigated the variability of $12.2-\mathrm{GHz}$ methanol masers detected towards MMB $6.7-\mathrm{GHz}$ sources in the $186^{\circ}-330^{\circ}$ longitude region, finding that the weaker $12.2-\mathrm{GHz}$ methanol masers showed higher levels of variability than the sources with higher peak flux densities. We similarly find evidence that the weaker $6.7-\mathrm{GHz}$ methanol masers show greater levels of variability. Table 3 shows the absolute percentage variations of the $6.7-\mathrm{GHz}$ methanol masers split into three peak flux density categories. The effect of noise in the lowest peak flux density category will be more 
Table 3. Mean and median variations in the $6668-\mathrm{MHz}$ methanol maser peak flux density split into three flux density categories (note that these are based on the survey cube and 'MX' measurements).

\begin{tabular}{lcc}
\hline $\begin{array}{l}\text { Flux density } \\
(\mathrm{Jy})\end{array}$ & $\begin{array}{c}\text { Mean variation } \\
\text { (per cent) }\end{array}$ & $\begin{array}{c}\text { Median variation } \\
\text { (per cent) }\end{array}$ \\
\hline Peak $<1.5$ & 35.9 & 21.3 \\
$1.5 \geq$ peak $<5$ & 26.5 & 19.1 \\
Peak $\geq 5$ & 19.6 & 14.2 \\
\hline
\end{tabular}

significant, accounting for a 10 per cent variation simply for $1 \sigma$ survey rms noise on a 1.5-Jy signal. In comparison, for the middle category, the $1 \sigma$ survey rms noise will contribute between 3 per cent and 10 per cent variation (and for the highest category, it will contribute $\leq 3$ per cent). Caswell et al. (1995) compared the fraction of variable spectral features in $2456668-\mathrm{MHz}$ masers, divided into four equal groups, ranked by flux density, and found no appreciable difference between the groups. While this appears to be contrary to our result, the Caswell sample, while large, was a result of targeted observations towards $\mathrm{OH}$ masers and was therefore biased towards more evolved sources with much higher peak flux densities (median of $19 \mathrm{Jy}$ ) compared to the MMB (median of $1.5 \mathrm{Jy}$ in the survey observations). Due to this, their sample does not have a comparable population of weak $6.7-\mathrm{GHz}$ methanol masers to show greater levels of temporal variations.

At least 186 668-MHz methanol masers have now been identified as showing periodic variations (Goedhart, Gaylard \& van der Walt 2003, 2004; Goedhart et al. 2009, 2014; Araya et al. 2010; Szymczak et al. 2011; Fujisawa et al. 2014b,a; Maswanganye et al. 2015, 2016; Szymczak, Wolak \& Bartkiewicz 2015). We have only a few epochs of observations and so we can speculate that only our most variable sources could be potential candidates for future monitoring for periodicity. However, since none of the 15 known periodically variable sources that fall within the longitude range of the MMB observations varied by more than a factor of 2 in our observations, this is clearly not a very efficient way of isolating the population of periodically varying sources. Green et al. (2010) highlighted $14.230-0.509$ and $15.607-0.255$ as particularly promising candidates for variability studies, noting their tendency to increase and decrease in peak flux density over the four epochs when they were observed.

\section{GALACTIC DISTRIBUTION OF 6668-MHZ METHANOL MASERS}

In this section, we examine the Galactic distribution of methanol maser sources, initially in the context of the distance-independent quantities of longitude, latitude and velocity, and then utilizing the distances determined in Section 3. In addition to the full survey results, we also pay particular attention to the longitude range of $\pm 60^{\circ}$, where we have comparable sensitivity about the Galactic Centre.

\subsection{Longitude-latitude distribution}

Fig. 9 shows the distribution (number count) of 6668-MHz methanol masers with Galactic longitude combined with the distribution in Galactic latitude. The longitude distribution peaks overall at $|l| \approx$ $30^{\circ}$, but at positive longitudes, the actual peak is at $30^{\circ}-35^{\circ}$, and at negative longitudes, it is at $20^{\circ}-25^{\circ}$. Note that the $35^{\circ}-40^{\circ}$ and $40^{\circ}-45^{\circ}$ bins show a higher number of known sources compared to the MMB (49 versus 35 and 34 versus 28, respectively). This is due to these regions having been observed to higher sensitivities by Pandian et al. (2007) and Olmi et al. (2014), where a total of 19 sources were detected with flux densities below the MMB $3 \sigma$ detection threshold and one source comparable to the threshold. Individual sources are listed in Breen et al. (2015). The folded distribution is shown in Fig. 10.

The latitude distribution is tightly constrained to the plane with an approximately Gaussian distribution with a full width at halfmaximum (FWHM) of $0.54 \pm 0.01$ and a mean latitude of -0.04 \pm 0.01 (Fig. 11). The width is comparable to the $0.5 \mathrm{FWHM}$ found by Pestalozzi et al. (2005), and the mean latitude implies that there is no significant bias in the latitude distribution, although there is a minor tendency to negative latitudes, as seen in other tracers (e.g. Urquhart et al. 2011; Contreras et al. 2013) and likely a result of the Sun's displacement relative to the Galactic plane. The distribution is also comparable to that of $\mathrm{H}$ II regions, which has been shown to have an FWHM of $0.61 \pm 0.04$ and a mean latitude of $-0.09 \pm$ 0.02 (Anderson et al. 2011).

\subsubsection{Implication for total population}

The MMB detection rate for the southern region of $186^{\circ}<l<300^{\circ}$ was 0.12 sourcesdeg $^{-2}$, implying that $\sim 60$ sources exist to the MMB sensitivity in the as-yet-unobserved northern region $\left(60^{\circ}<l<186^{\circ}\right)$. As a simple estimate, this would imply a total population of 1032 masers, which, factoring in the completeness estimate (Green et al. 2009a), would imply a true total population of $\sim 1290$ masers (with a peak flux density $>3 \sigma_{\text {rms }}$ ). A caveat is that the unobserved northern region includes the local Orion-Cygnus arm, which may produce a bias between the second and third Galactic quadrants through an increased sensitivity to lower luminosities (with high-mass star-forming regions within $1 \mathrm{kpc}$ ).

\subsection{Longitude-velocity distribution}

Fig. 4 shows the longitude-velocity distribution for the inner $60^{\circ}$ of Galactic longitude, overlaid with the commonly adopted logarithmic spiral arm model (Taylor \& Cordes 1993, transferred to the longitude-velocity domain through a flat rotation curve with a circular rotation of $246 \mathrm{~km} \mathrm{~s}^{-1}$ and a Galactocentric solar distance of $8.4 \mathrm{kpc}$ ). This distribution highlights that the peaks seen in the Galactic longitude distribution correspond to small regions in longitude-velocity space, rather than simply higher densities across all velocities. However, in contrast, the range of velocity over which emission is seen for an individual source tends to be wider for sources within these higher density regions (see Fig. 12, which shows the distribution of the range of velocities over which emission is seen from individual sources, for given longitude bins). This behaviour corresponds to the distribution of velocity ranges and detection rates with longitude for the associated 12-GHz maser population (see fig. 6 of Breen et al. 2016), and could be an indication that the more kinematically perturbed regions harbour higher star formation rates (and vice versa), although this requires corroboration with other tracers of the cloud kinematics.

The sources in the inner $20^{\circ}$ of Fig. 4, which are unassociated with spiral arm loci, have been shown (Green et al. 2009b, 2010, 2011) to be associated with a combination of the $3-\mathrm{kpc}$ arms (those in approximately linear distributions passing through +50 and $-50 \mathrm{~km} \mathrm{~s}^{-1}$ at $0^{\circ}$ longitude), the long Galactic bar (extreme positive velocities within this longitude range, including 7.632-0.109, with the highest 


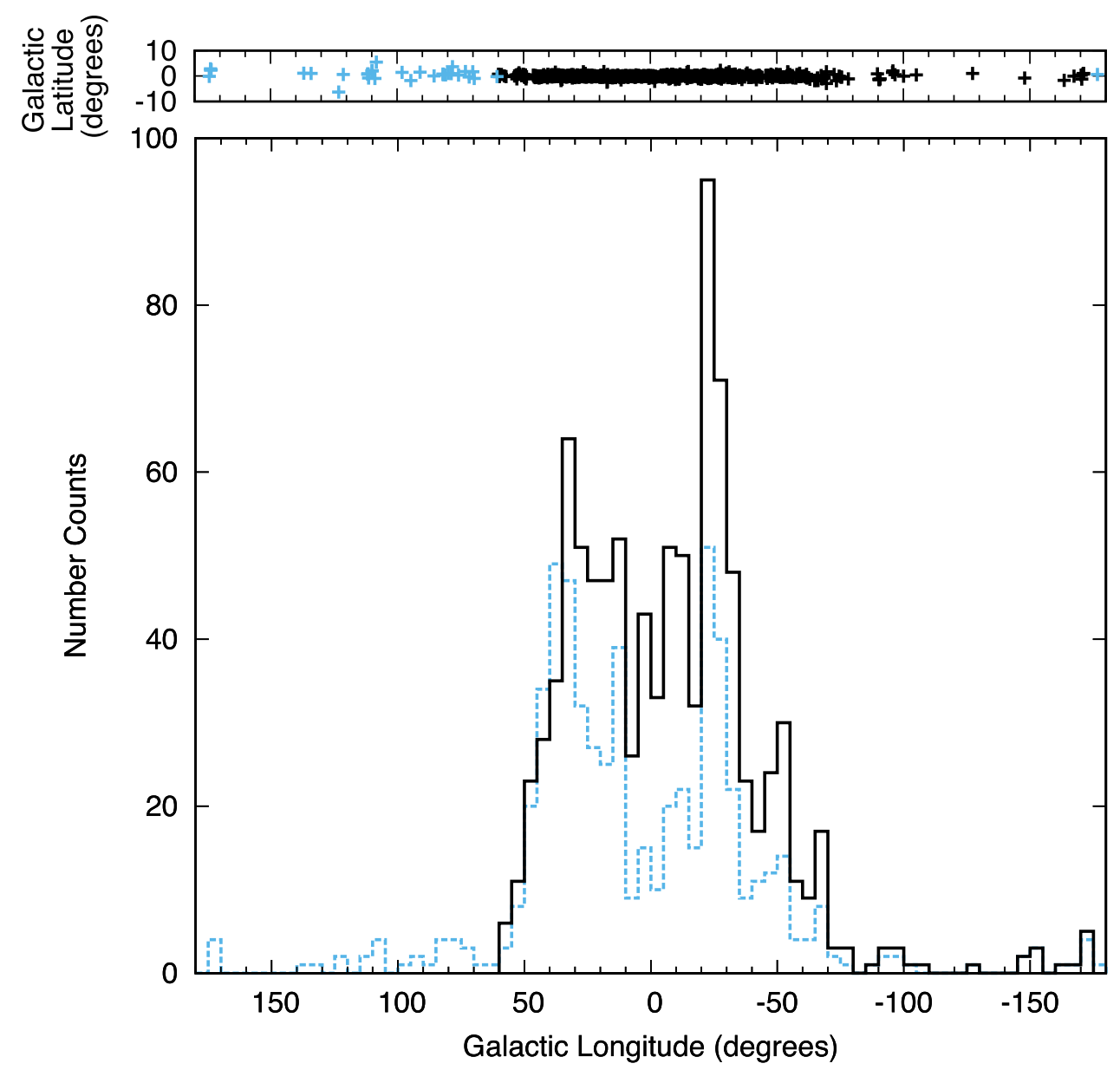

Figure 9. Top panel: longitude-latitude distribution of 6668-MHz methanol masers as detected by the MMB survey. Blue crosses show known sources outside the MMB survey region (see Pestalozzi et al. 2005; Ellingsen 2007; Pandian et al. 2007; Cyganowski et al. 2009, for details). Bottom panel: longitude distribution of $6668-\mathrm{MHz}$ methanol masers as detected by the MMB survey (solid) and those that were known prior to the MMB (dotted), in $5^{\circ}$ bins. The $35^{\circ}-40^{\circ}$ and $40^{\circ}-45^{\circ}$ bins show a higher number of known sources compared to the MMB as these regions incorporate the observations by Pandian et al. (2007) and Olmi et al. (2014), where sources were detected with flux densities below the MMB detection threshold.

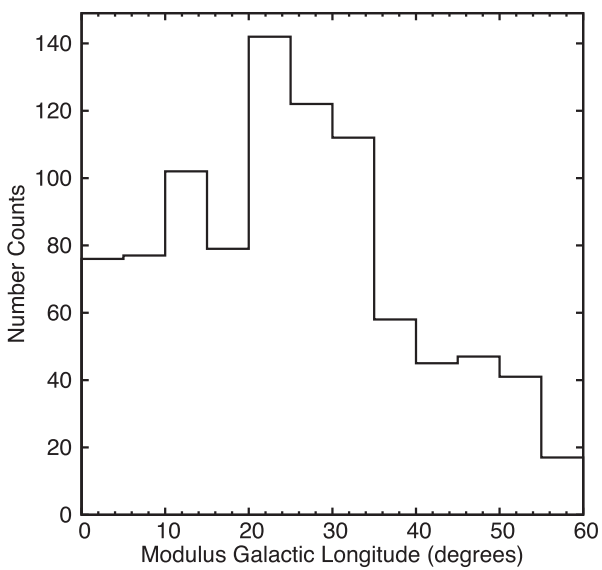

Figure 10. Folded longitude distribution of the MMB, corresponding to the number counts within $|60|^{\circ}$

peak velocity of $157 \mathrm{~km} \mathrm{~s}^{-1}$ ) and the Galactic Centre (specifically $\mathrm{Sgr}$ B2, with the sources at a range of velocities at $\sim 0.6$ longitude). This association has highlighted that there is ongoing HMSF in these structures (Green et al. 2009b, 2011). The range of individual velocities (Fig. 12) shows a higher number of sources with small

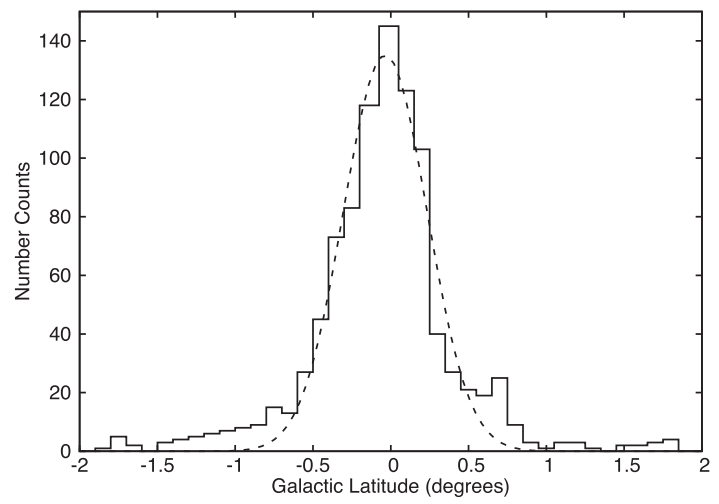

Figure 11. Latitude distribution of the MMB detections with Gaussian fit. Mean latitude is $-0.04 \pm 0.01$ and FWHM is $0.54 \pm 0.01$.

velocity ranges $\left(\leq 5 \mathrm{~km} \mathrm{~s}^{-1}\right)$ for the longitude bins that include the 3 -kpc arms, $\left( \pm 20^{\circ}\right)$. The presence of spiral arm tangents influences the $20^{\circ}-30^{\circ}$ and $-20^{\circ}$ to $-30^{\circ}$ bins, with the Norma and Perseus arm origins also contributing to the $-20^{\circ}$ to $-30^{\circ}$ longitude bin, bringing a higher propensity of sources with wider velocity ranges (of the order of $10 \mathrm{~km} \mathrm{~s}^{-1}$ ). 


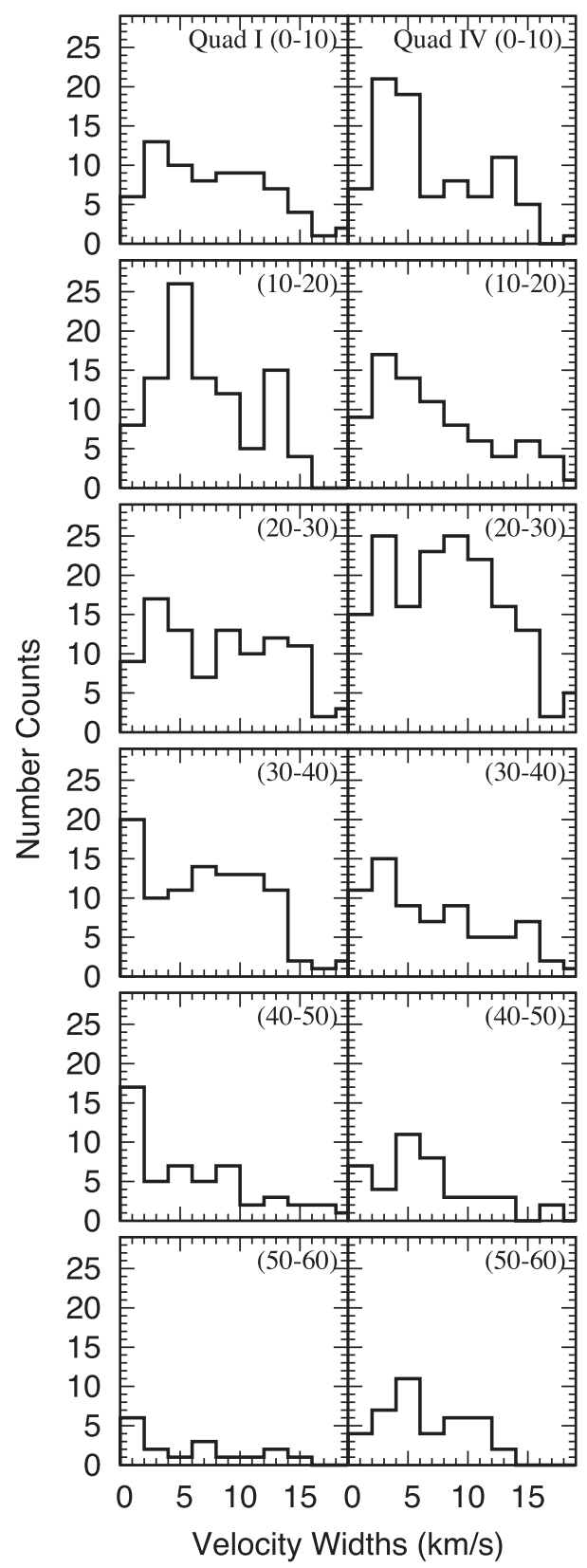

Figure 12. Distribution of velocity widths split by $10^{\circ}$ intervals of Galactic longitude for the first and fourth quadrants of the Galaxy.

Previously, Green et al. (2011) explored the density of masers in longitude-velocity space for the inner $28^{\circ}$ through calculating a second-order structure function of maser velocity as a function of angular separation between maser sites. This involved creating a reference Monte Carlo simulation of randomly distributed masers, within the longitude range, and with velocities matching Galactic rotation (on the basis of a solar distance of $8.4 \mathrm{kpc}$ and a flat rotation curve with a circular velocity of $246 \mathrm{~km} \mathrm{~s}^{-1}$ ). This simulation could then be compared with the real sample to determine if there was significant structure in the distribution (where a zero gradient fit would indicate no scale-specific structure). The study found a small positive gradient in the structure function of the real sample, departing from the flat structure function of the simulation for separations less than 0.03 and velocities up to $30 \mathrm{~km} \mathrm{~s}^{-1}$, and at scales greater than $3^{\circ}$ and velocities up to $50 \mathrm{~km} \mathrm{~s}^{-1}$, essentially indicating correlation on small scales and clustering of structure on large scales. This meant that the maser density data for the inner $28^{\circ}$ could be smoothed on the small scales and binned on the large scales to produce a density profile.

For the current sample of $\pm 60^{\circ}$, if we adopt the same approach as Green et al. (2011), we find that although the structure function fits are comparable to the previous, the larger source count, and greater range of longitudes and velocities (and variation of those with longitude), we do not find statistically significant separations between simulation and real samples. As such for this larger sample, we cannot simply bin the sources in the longitude-velocity domain when constructing a density distribution, and we simply present the distribution as is in Fig. 13. We see evidence for increased number counts towards the origins of all four spiral arms and the interaction of the (long) Galactic bar and the 3-kpc arm tangent (Fig. 13). Additionally, Fig. 13 shows higher densities at the Norma, CruxScutum and Carina-Sagittarius spiral arm tangents. This behaviour is also seen in the associated $12-\mathrm{GHz}$ methanol maser population (Breen et al. 2016).

\subsubsection{Comparison with atomic and molecular tracers}

Fig. 14 shows the masers overlaid on the $\mathrm{H}_{\mathrm{I}}$ emission of the Parkes Galactic All Sky Survey (GASS; McClure-Griffiths et al. 2009) for $-60^{\circ}$ to $\sim 34^{\circ}$ and VGPS (Stil et al. 2006) for $\sim 34^{\circ}-60^{\circ}$, with the latter survey data spatially smoothed to the GASS resolution. The masers are found to lie within the extreme envelope of the $\mathrm{H}$ emission with all sources within $10 \mathrm{~km} \mathrm{~s}^{-1}$ of the terminal velocity with the exception of one source in the first Galactic quadrant, $33.486+0.040$, with a peak velocity of $122.8 \mathrm{~km} \mathrm{~s}^{-1}$, which is likely to be a source subject to a large peculiar velocity $\left(\sim 20 \mathrm{~km} \mathrm{~s}^{-1}\right.$ offset from the nearest young stellar object in the MSX catalogue; Lumsden et al. 2013). The fact that almost all the sources of the MMB lie within the H I envelope indicates that the masers follow the gas distribution and are not subject to large anomalous velocities. There is a lack of maser emission in the more extreme outer Galaxy velocities of $\mathrm{H}_{\mathrm{I}}$ emission, particularly noticeable for longitudes $-60^{\circ}$ to $-30^{\circ}$ and velocities exceeding $50 \mathrm{~km} \mathrm{~s}^{-1}$, believed to be associated with the far side of the Perseus arm. We would expect methanol masers to be associated with the spiral arms on the basis of their exclusive relation with HMSF, but at these large Galactocentric distances, the metallicity (and, by implication, the methanol abundance) of the Galaxy is known to drop significantly (e.g. Balser et al. 2011; Cheng et al. 2012), and this could directly impact the conditions for maser emission, most likely by reducing the flux density below the sensitivity limit of the MMB. The masers also broadly trace the $\mathrm{H}_{\mathrm{I}}$ clumps within the distribution shown in the integrated emission of Fig. 14, with a few notable exceptions: high (positive) velocity Galactic Centre masers not significantly traced in $\mathrm{HI}$; masers towards the terminal velocity between $20^{\circ}$ and $30^{\circ}$ Galactic longitude; and masers associated with $\mathrm{H}_{\mathrm{I}}$ emission between $0^{\circ}$ and $-25^{\circ}$ Galactic longitude with velocities exceeding $-50 \mathrm{~km} \mathrm{~s}^{-1}$. All these regions have much narrower $\mathrm{H}_{\mathrm{I}}$ emission profiles, which are effectively smoothed out in the integrated plot. The H I emission profiles can demonstrate a peak either due to velocity fluctuations or increased density, and the broad association of masers with clumps would indicate that density is the dominant factor. The association also confirms that the bright/dense $\mathrm{H}$ I emission generally traces the spiral arms.

Fig. 15 shows the masers overlaid on the $\mathrm{CO}$ emission of Dame, Hartmann \& Thaddeus (2001), where it is clear that the maser 


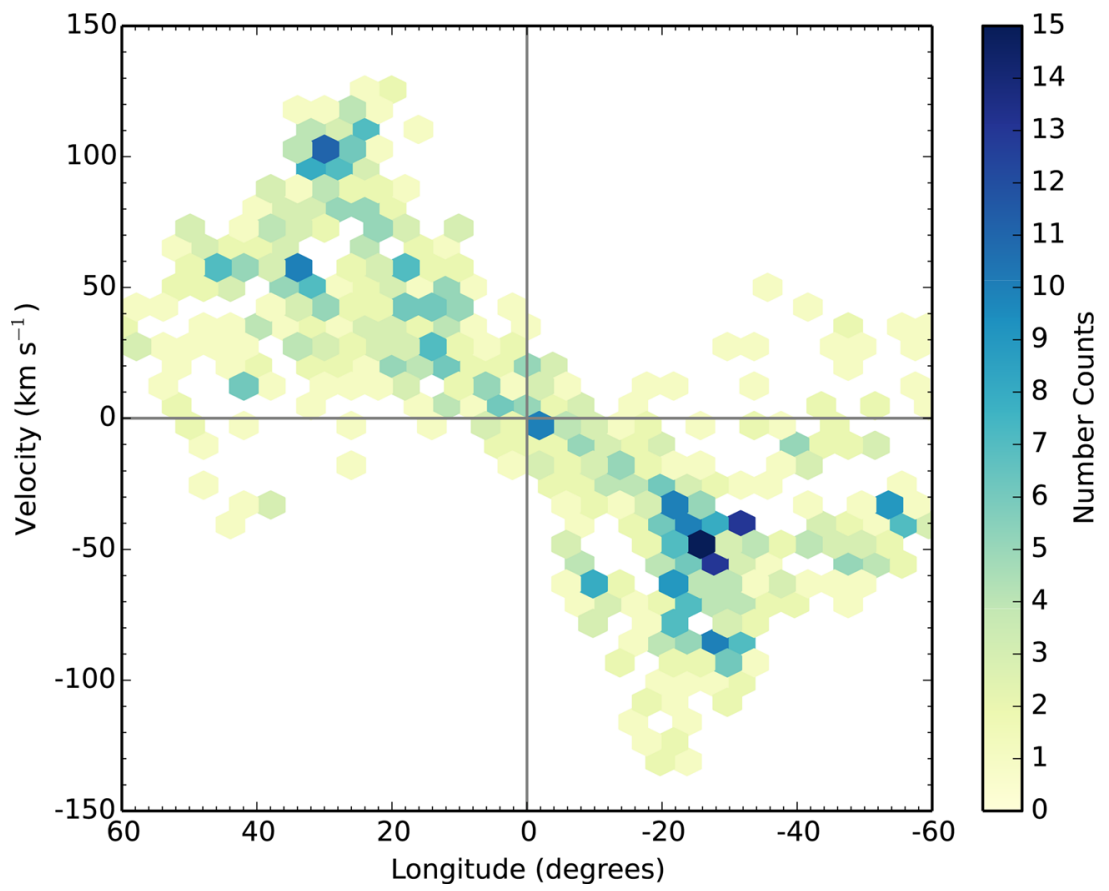

Figure 13. Longitude-velocity density distribution for inner $60^{\circ}$ of Galactic longitude. In comparison with Fig. 4, pockets of higher density are seen at the tangents of the spiral arms and the arm origins.

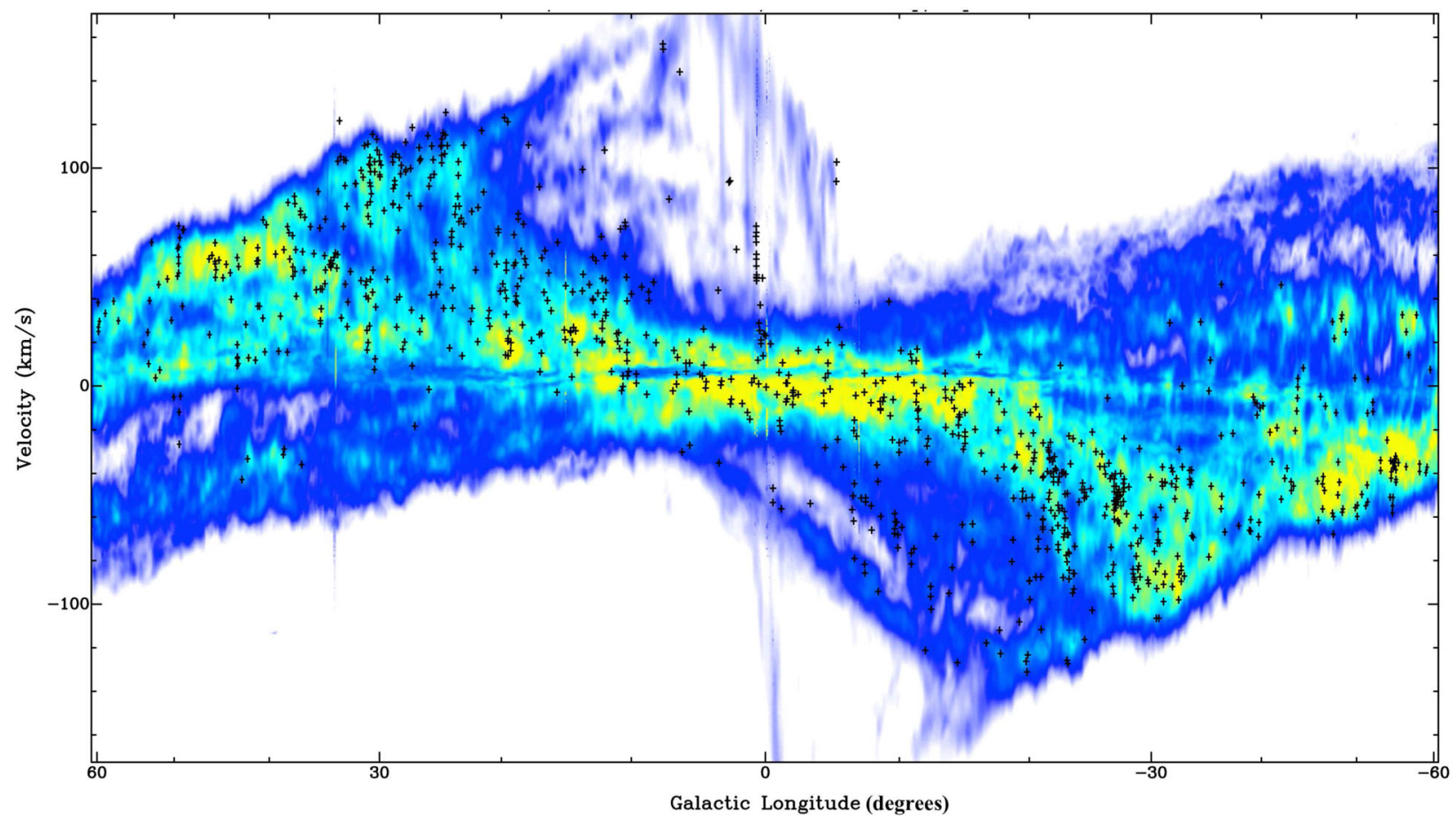

Figure 14. 6668-MHz methanol masers of the MMB overlaid on the $\mathrm{H}$ i emission of the GASS and VGPS data sets (southern and northern longitudes, respectively), smoothed to GASS resolution, with emission averaged between Galactic latitudes $b=-1^{\circ}$ and $+1^{\circ}$.

distribution, similar to the $\mathrm{H}_{\mathrm{I}}$ comparison, broadly traces the brighter emission, and thus denser clumps, confirming like $\mathrm{H}_{\mathrm{I}}$, that the dense $\mathrm{CO}$ emission traces the spiral arms. However, for far-side Galactic structures (negative velocities in the first Galactic quadrant and positive in the fourth Galactic quadrant), in contrast to the $\mathrm{H}_{\mathrm{I}}$ where we see emission without masers, here we find a number of maser sources that are unassociated with $\mathrm{CO}$. However, this is likely to be a result of the $\mathrm{CO}$ emission associated with molecular clouds located at the far side of the Galaxy falling below the detection threshold. Also note that for the extreme range of velocities (both positive and negative) exhibited towards the Galactic Centre and Central Molecular Zone, it appears in Fig. 15 that the positive 


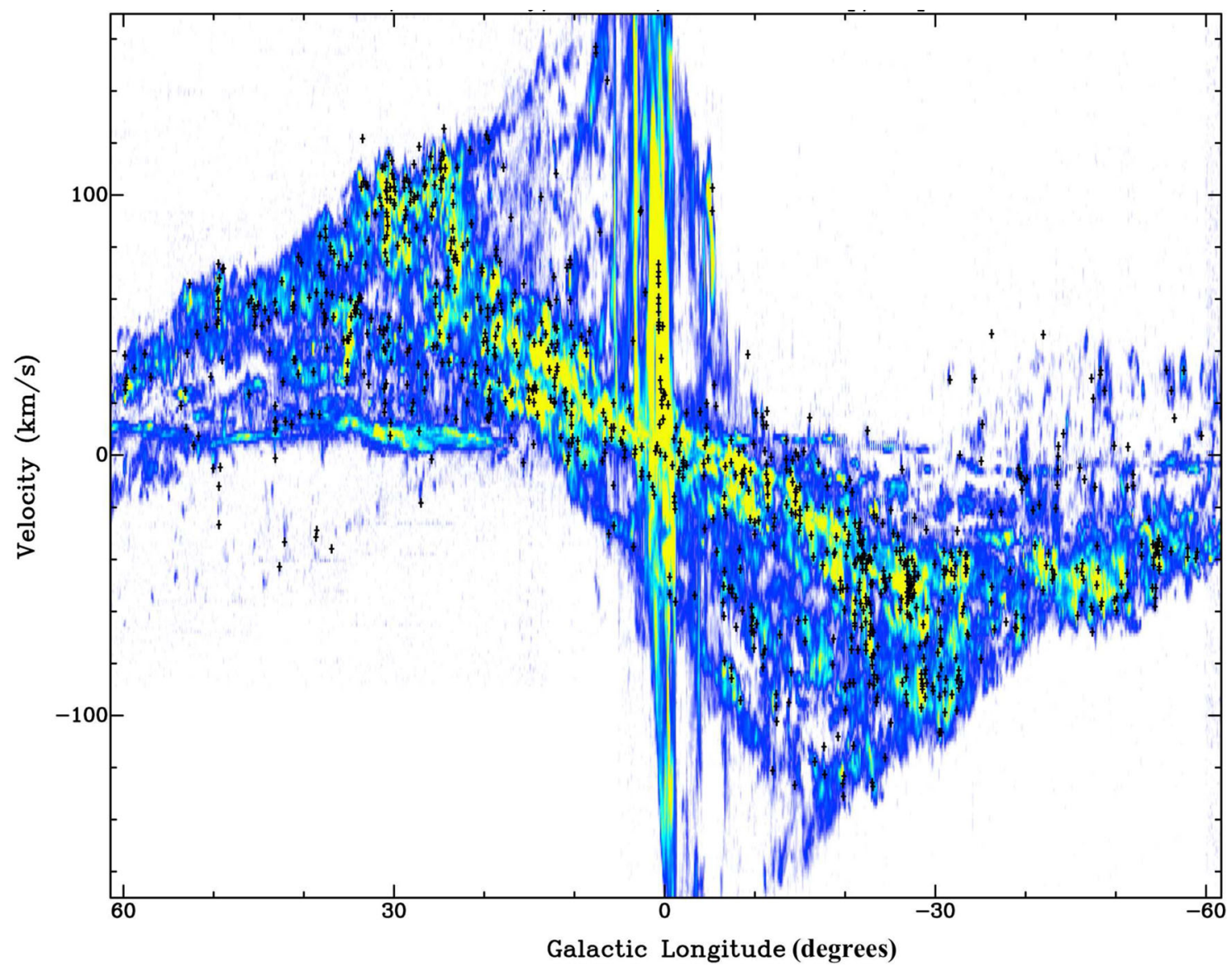

Figure 15. 6668-MHz methanol masers of the MMB overlaid on the CO (1-0) emission of Dame et al. (2001), summed between Galactic latitudes $b=-1^{\circ}$ and $+1^{\circ}$.

velocities are favoured, with predominantly more sources associated with these; however, this is biased by the 11 sources associated with Sgr B2.

A separate paper is exploring the association with the molecular (ammonia) emission, together with other maser species, detected in the HOPS survey (Breen et al., in preparation), but Fig. 4 includes lines indicating arm tangents and origins from the study of multiple tracers of Vallée (2016). It is worth noting that although the methanol masers align well with the other tracers, and through their association with HMSF will trace the spiral arms, Fig. 4 highlights that the arm loci of models have their limitations. Although the assumption of logarithmic spirals is broadly acceptable, they do not trace well on the smaller scales (where inference dominates data), with a number of dense regions/masers that do not fall on arm loci. A complete census of astrometric measurements will solve this.

\subsection{Galactic distribution through kinematic distances}

To examine the Galactic distribution of $6668-\mathrm{MHz}$ methanol masers, we utilize the distances described in Section 3. As noted, these are primarily kinematic resolutions, with some astrometric measurements, and in total, we have distances for 778 sources. The distribution is shown in Fig. 16. We find that the median (heliocentric) distance is $5.6 \mathrm{kpc}$ and there are 15 sources with distances exceeding $15 \mathrm{kpc}$, with the farthest source being $343.929+0.125$ with a distance of $18.6 \pm 1.3 \mathrm{kpc}(323.766-1.370$ is calculated to have a slightly larger distance of $18.7 \pm 1.1 \mathrm{kpc}$, but the large

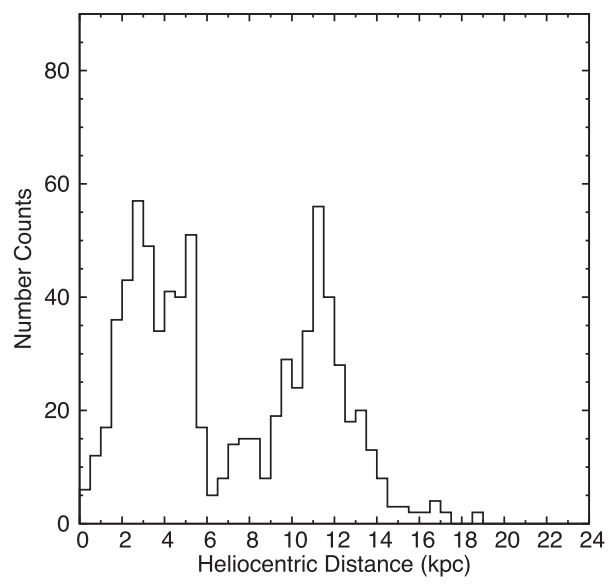

Figure 16. Distribution of number counts with heliocentric distances.

latitude of this source would suggest a near distance, as it would otherwise be $\sim 450 \mathrm{pc}$ from the plane, which is well beyond the scaleheight of the masers).

The Galactocentric distances have the benefit of not relying on the resolution of the distance ambiguity problem. However, they do suffer a loss of positional information and are still subject to the accuracy of the kinematic model. We find a median Galactocentric distance of $5.5 \mathrm{kpc}$, and three sources exceeding $12 \mathrm{kpc}$. The overall Galactocentric distribution is shown in Fig. 17. A suggestion of a peak at $\sim 4.5 \mathrm{kpc}$ is seen, and additionally at $\sim 6 \mathrm{kpc}$, which is similar 


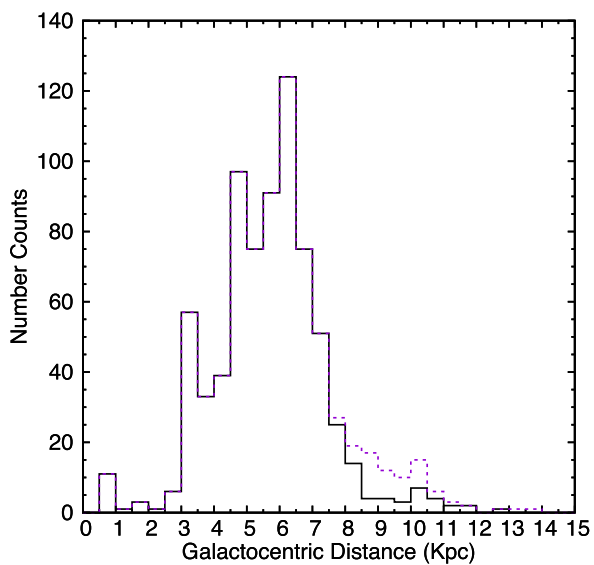

Figure 17. Galactocentric distribution using standard kinematic distances and H ISA for sources within $\pm 60^{\circ}$ longitude (solid black) in 0.5 -kpc bins. The dashed purple line shows the additional sources that exist in the $-60^{\circ}$ to $-180^{\circ}$ longitude range (all in excess of $7.5 \mathrm{kpc}$ ). Beyond $R=12 \mathrm{kpc}$, there are only three sources, with Galactocentric radii of 12.9, 13.2 and $13.7 \mathrm{kpc}$.

to those seen in $\mathrm{H}$ II regions (Anderson et al. 2012) and mid-infrared sources associated with HMSF (Urquhart et al. 2014a).

The number counts for Galactocentric distances are broken down by longitude ranges in Fig. 18, highlighting different Galactic structures influencing the number counts for different longitude bins. We see individual peaks tied to the spiral arms, particularly along the tangents of the spiral arms, which fold into the overall picture seen in Fig. 9. This is to be expected, as the masers trace HMSF, which is known to be tied to the spiral arms, and so when there is a greater length of spiral arm along the line of sight, there will be higher numbers of masers. The asymmetry in longitude is likely due to the orientation and position of spiral arm features, with the asymmetry mirrored in our understanding of Galactic structure, specifically the orientation and interaction of a long Galactic bar (Hammersley et al. 2000; Benjamin et al. 2005; Cabrera-Lavers et al. 2008). Here, we discuss each of the longitude bins:

(i) For the $\mid 0^{\circ}$ to $10^{\circ} \mid$ region, identifying structures is difficult due to the number of structures present (all spiral arms cross the line of sight, plus numerous Galactic Centre features) and the uncertainty that arises for velocities close to $0 \mathrm{~km} \mathrm{~s}^{-1}$ (where the uncertainty in velocity can have a large impact on distance, and has led to their exclusion from our kinematic distance allocations).

(ii) For the $\left|10^{\circ}-20^{\circ}\right|$ region, the association with the $3-\mathrm{kpc}$ arms/ring is prominent, as to is the Perseus arm in both quadrants.

(iii) For the $\left|20^{\circ}-30^{\circ}\right|$ region, the Perseus and Norma arms contribute to a 5-6 kpc peak in the fourth quadrant, with Norma tangential to the line of sight, and the Perseus and Crux-Scutum arms contribute to a $<5 \mathrm{kpc}$ and $\sim 7 \mathrm{kpc}$ peak in the first quadrant, respectively.

(iv) For the $\left|30^{\circ}-40^{\circ}\right|$ region, the Perseus arm continues, the Carina-Sagittarius arm comes in and there is a hint of the Norma arm in the first quadrant, whilst in the fourth quadrant, we move beyond the Perseus and Norma arm influence and see the Crux-Scutum arm and distant components of the Carina-Sagittarius arm at $\sim 10 \mathrm{kpc}$.

(v) For the $\left|40^{\circ}-50^{\circ}\right|$ region, the Carina-Sagittarius arm at $\sim 6 \mathrm{kpc}$ and the Perseus arm at an $\sim 7 \mathrm{kpc}$ are evident for the first quadrant, and the Crux-Scutum arm at $\sim 6 \mathrm{kpc}$ together with the continuing distant components of the Carina-Sagittarius arm at $\sim 10 \mathrm{kpc}$ are seen for the fourth quadrant.

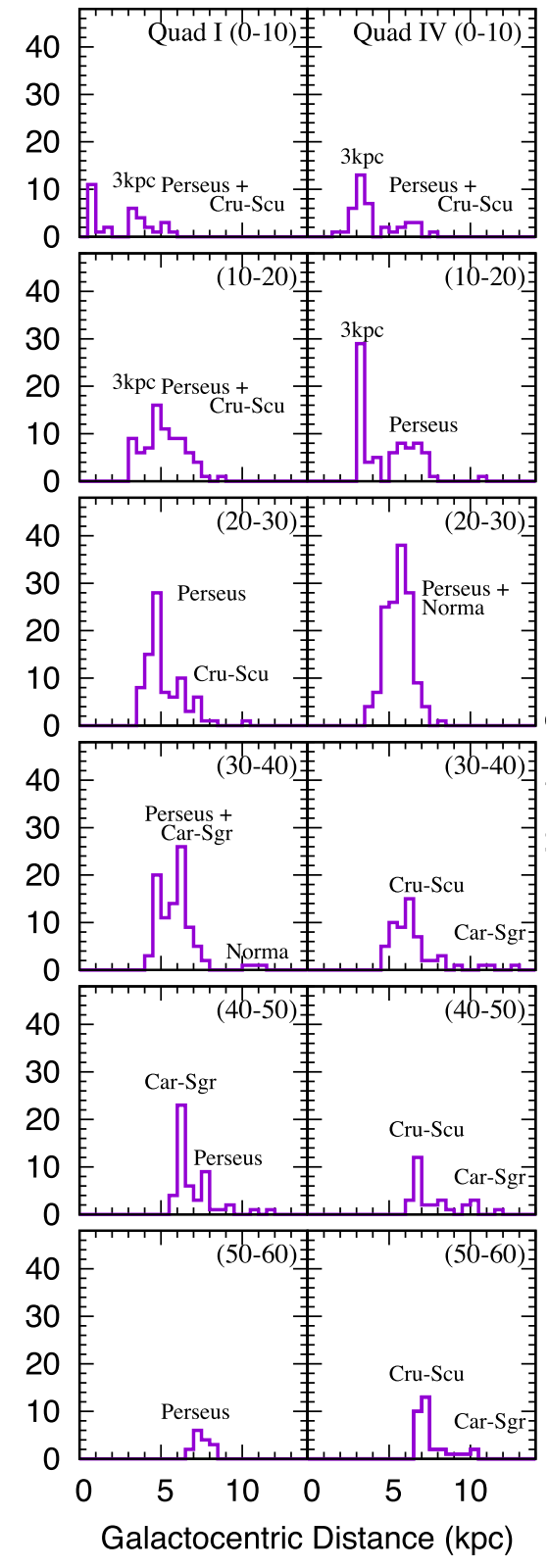

Figure 18. Galactocentric distribution split by $10^{\circ}$ intervals for the first and fourth Galactic quadrants with labels identifying likely corresponding structures. Within $10^{\circ}$ of the Galactic Centre, the key features are the 3$\mathrm{kpc}$ arms/ring, the Perseus and Crux-Scutum arms at $\sim 5 \mathrm{kpc}$ and a slight influence of the Norma arm at $7 \mathrm{kpc}$.

(vi) And for the $\left|50^{\circ}-60^{\circ}\right|$ region, the Perseus arm almost exclusively contributes an $\sim 7 \mathrm{kpc}$ peak to the first quadrant, the CruxScutum arm a similar $\sim 7 \mathrm{kpc}$ peak to the fourth quadrant, and distant components of the Carina-Sagittarius arm remain present at $\sim 10 \mathrm{kpc}$.

\subsection{Vertical structure and scaleheight}

The distribution of distance from the Galactic plane, $z$, is shown in Fig. 19. A Gaussian fit reveals an FWHM of the distribution of 56.5 $\pm 3.2 \mathrm{pc}$. 


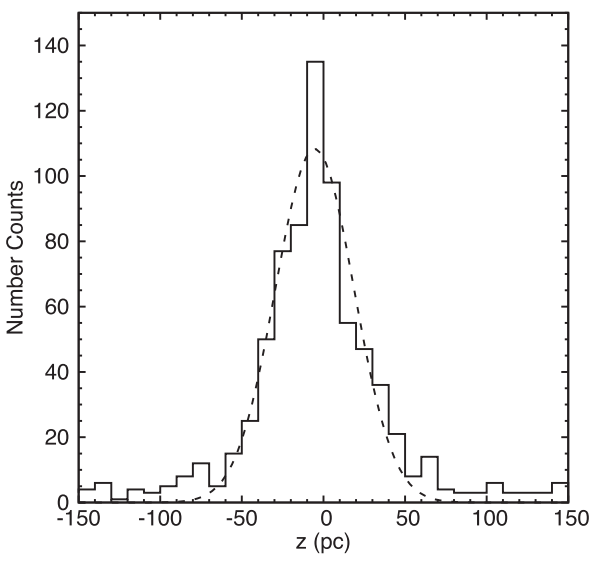

Figure 19. Distribution of height from the Galactic plane with a Gaussian fit; FWHM is $56.5 \pm 3.2 \mathrm{pc}$.

If the distribution is folded, $|z|$, and fitted with an exponential, the scaleheight is found to be $26.6 \pm 1.6 \mathrm{pc}$.

This is within the errors of that found for the smaller sample of Green \& McClure-Griffiths (2011), and comparable to those found previously for HMSF (see discussion of Green \& McClureGriffiths 2011), and those found recently (e.g. Urquhart et al. 2014a, for comparable longitudes).

\subsection{Luminosity distribution}

Maser emission results from direction-dependent beaming; however, statistically, the emission from beamed maser spots should appear the same from all directions (Elitzur 1992). Therefore, we calculate the maser luminosity, assuming the emission is isotropic. We utilize the integrated flux density of the maser emission, as presented in Breen et al. (2016), with the distances determined in Green \& McClure-Griffiths (2011) and the current HiSA work, to determine the maser luminosity. Taking into account those sources that either did not have a distance estimate or for which an integrated flux density could not be determined resulted in a sample of 732 sources. We first discuss the luminosities as a product of the integrated flux density and distance squared, and then as a function of solar luminosities with the global assumption of a linewidth of $5.56 \mathrm{kHz}$ (velocity of $0.2 \mathrm{~km} \mathrm{~s}^{-1}$; Pestalozzi et al. 2007), presenting the result in Fig. 20. The two luminosity distributions are of course effectively scaled versions of one another (hence only the latter is shown). This can be compared with the estimate of the hydroxyl maser luminosity function presented in Caswell \& Haynes (1983), which has of the order of 100 sources at $100 \mathrm{Jy} \mathrm{kpc}^{2}$, and predicts a rise to almost 1000 sources at $<10 \mathrm{Jy} \mathrm{kpc}^{2}-$ this would correspond to a continual rise below the MMB $3 \sigma$ sensitivity at the edge of the Galaxy (blue vertical line in Fig. 20). A power-law fit above

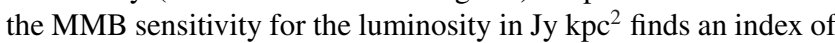
$-0.32 \pm 0.04$ (note that this is a shallower index than reported in Green \& McClure-Griffiths 2011 as a lower luminosity, courtesy of the more complete sample, is taken for the boundary of the fit).

The luminosity distribution derived here can be used to examine the expectations for the populations of nearby galaxies. For the Large Magellanic Cloud (LMC), four sources have been detected, with luminosities exceeding $500 \mathrm{Jy} \mathrm{kpc}^{2}$ (Green et al. 2008), one in M31 with a luminosity of $5120 \mathrm{Jy} \mathrm{kpc}^{2}$ (Sjouwerman et al. 2010), and a limit was placed on NGC253 of $\sim 1.3 \times 10^{6} \mathrm{Jy} \mathrm{kpc}^{2}$ (Phillips et al. 1998). For these three respective limits, the Galactic population

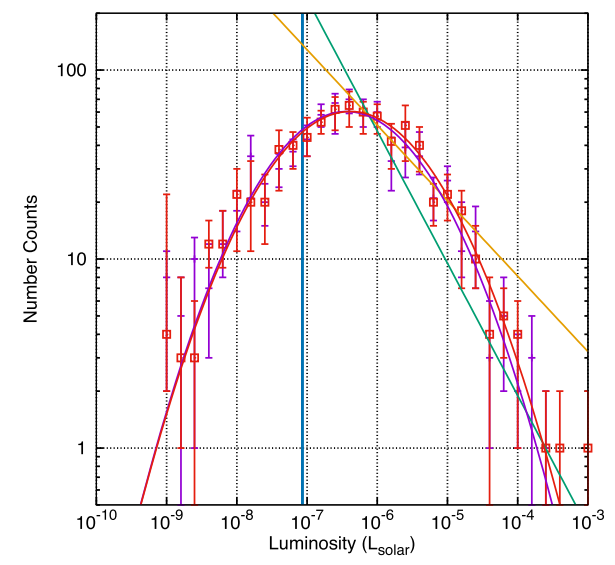

Figure 20. Distribution of maser luminosity with lognormal fits (solid red and purple lines). Errors represent both the kinematic distance errors and the statistical uncertainty. Purple crosses and lines are the standard kinematic distances (and for comparison, red squares and lines are the Reid et al. 2016 distances discussed in Section 3.3, highlighting the similarity of the resultant luminosity distributions). The blue vertical line shows the MMB $3 \sigma$ sensitivity at a distance of $22 \mathrm{kpc}$ heliocentric distance. The amber line shows a power-law fit to sources above the peak at $10^{-6.5}$, and the green line indicates the power-law index of the previous Pestalozzi et al. (2007) study.

using the HiSA distances is 211,45 and 0 , and using the Reid et al. (2016) distances, it is 235, 49 and 0 . We therefore cannot make any conclusions with regards to NGC253, but we can say that both the LMC and M31 populations are 45-50 times smaller than the Galactic populations. For the LMC, this is the same conclusion as found by Green et al. (2008) in comparison with the smaller Pestalozzi et al. (2007) catalogue luminosity distribution.

In terms of solar luminosities, we find the number counts peak in the region of $\sim 10^{-6.5} \mathrm{~L}_{\odot}$ (the median luminosity is $2.7 \times 10^{-7} \mathrm{~L}_{\odot}$ ), and has a broadly Gaussian distribution in the $\log -\log$ plane. The peak is comparable to the peak of the predicted distribution of Pestalozzi et al. (2007), which was based on a model for a 2-Jy survey sensitivity, but the MMB distribution differs, however, below the peak. The MMB distribution (with 0.17-Jy $1 \sigma$ survey sensitivity) has a Gaussian fall-off at lower luminosities rather than the rapid fall-off found in the previous (sensitivity limited) work.

With global assumptions of near/far distances, Pestalozzi et al. (2007) fitted a power law between luminosities of $10^{-8}$ and $10^{-3} \mathrm{~L}_{\odot}$, finding an index of -1.7 (equivalently -0.7 for equal logarithmic luminosity bins). A power law is clearly a very poor fit to the MMB luminosity over this same luminosity range. Above the MMB $3 \sigma$ sensitivity limit (at $22 \mathrm{kpc}$ ), we can fit a power-law index of $-0.25 \pm 0.04$, and if we fit purely above the peak at $\sim 10^{-6.5}$, we find an index of $-0.40 \pm 0.04$ (with a more palatable 10 per cent error margin, shown in Fig. 20).

We can instead choose to fit the whole distribution, to incorporate the turnover, using a least-chi-squares fit of the distribution with a lognormal form (a Gaussian in the logarithm of the independent variable, also referred to as a Galton distribution)

$f(x)=(a / x) \mathrm{e}^{-(\log (x)-b)^{2} / 2 c^{2}}$,

finding $a=0.00024 \pm 0.00006, b=-10.1 \pm 0.5$ and $c=2.18 \pm$ 0.11 , where the errors are the formal errors of the fits. The fits along with the errors incorporating the distance uncertainty together with the fit uncertainty can be seen in Fig. 20. The mode of the lognormal distribution, a measure of the peak of the distribution given by $\mathrm{e}^{b-c^{2}}$, is $3.5_{-2.2}^{+5.8} \times 10^{7} \mathrm{~L} \odot$. The skewness, given by $\left(\mathrm{e}^{c^{2}}+2\right) \sqrt{\left(\mathrm{e}^{c^{2}}-1\right)}$, 


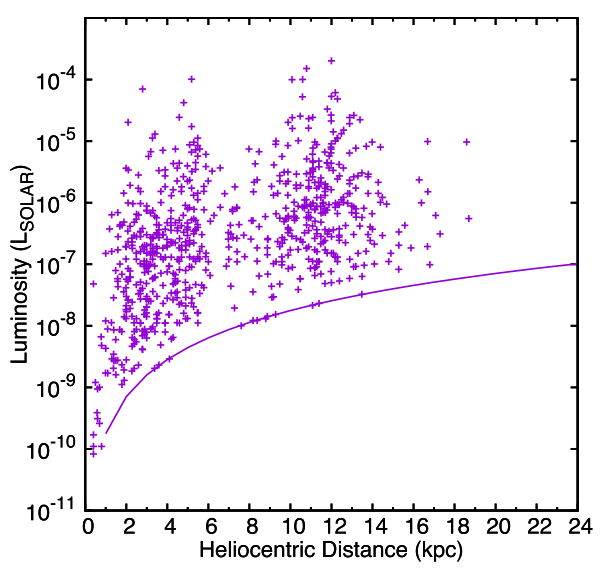

Figure 21. Luminosity versus heliocentric distance. The solid line represents the $3 \sigma$ sensitivity of the MMB.

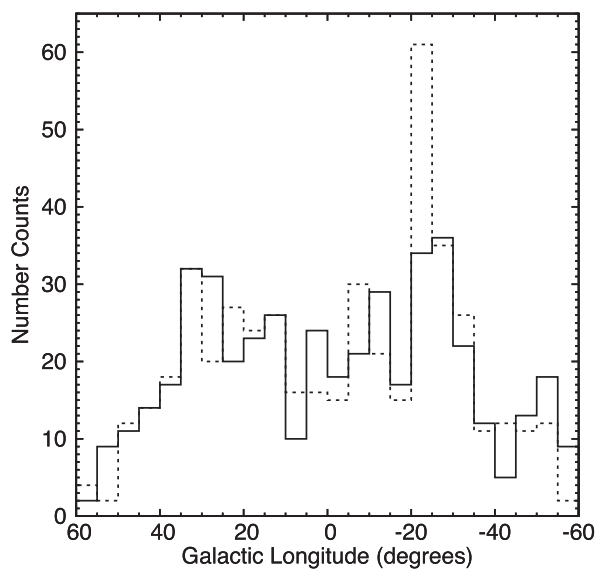

Figure 22. Longitude distribution differentiating sources with flux densities $<5.1 \mathrm{Jy}$ (solid line) and sources with flux densities $>5.1 \mathrm{Jy}$ (dashed) sources, where $5.1 \mathrm{Jy}$ is chosen to give a 50 per cent split between faint and bright sources. Overall distributions are largely comparable but there is a significant bright abundance at $-25^{\circ}$ corresponding to a dense cluster of sources in the longitude-velocity domain.

is $1263_{-632}^{+1365}$. Finally, Fig. 21 shows the luminosity distribution with heliocentric distance against the MMB sensitivity.

It is clear that the distribution significantly above the MMB sensitivity limit will not change with future more sensitive surveys, subject to source variability, but the question remains as to how the Galactic distribution may change below the limit (noting that the limit shown on Fig. 20 is conservative, located at the edge of the Galaxy at $22 \mathrm{kpc}$ ), for which deeper, more sensitive surveys are required (for example, with the Square Kilometre Array).

\subsubsection{Longitude-flux and longitude-luminosity distributions}

The distribution of source counts over the inner $60^{\circ}$ of Galactic longitude is first shown in Fig. 22 split based on the observable, flux density, as the brighter 50 per cent and the weaker 50 per cent (flux densities greater than or less than $5.1 \mathrm{Jy}$, respectively). The proportions are largely in agreement with the notable exception of a dominance of brighter sources in the $-20^{\circ}$ to $-25^{\circ}$ longitude bin. The distribution of source counts over the inner $60^{\circ}$ of Galactic longitude is then shown in Fig. 23 split based on the distance-derived luminosity, split either side of the peak of the luminosity distribution

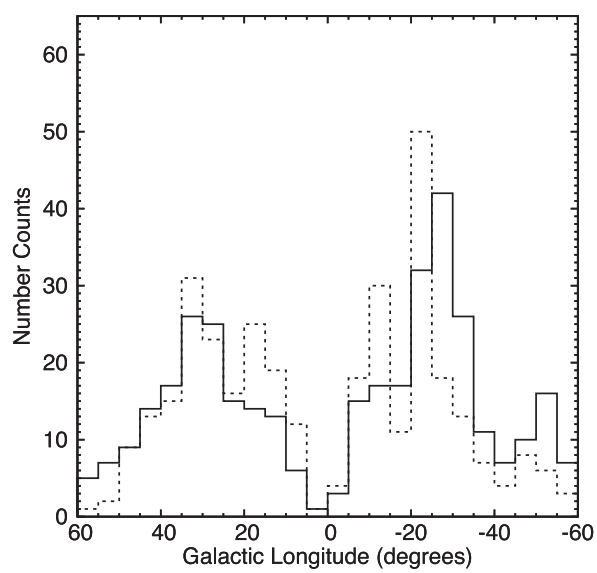

Figure 23. Longitude distribution differentiating sources with luminosities $<10^{-6.5} \mathrm{~L}_{\odot}$ (solid line) and sources with luminosities $>10^{-6.5} \mathrm{~L}_{\odot}$ (dashed) sources, where $10^{-6.5} \mathrm{~L}_{\odot}$ is the approximate peak in the luminosity distribution. There are significantly more brighter sources in the $-25^{\circ}$ to $-20^{\circ},-15^{\circ}$ to $-10^{\circ}$ and $15^{\circ}-20^{\circ}$ longitude bins.

described in the last section (less than and greater than $10^{-6.5} \mathrm{~L}_{\odot}$ ). Here again, there is a notable dominance of bright sources in the $-20^{\circ}$ to $-25^{\circ}$ longitude bin, but there are also significant abundances in the $-15^{\circ}$ to $-10^{\circ}$ and $15^{\circ}-20^{\circ}$ longitude bins.

The $-20^{\circ}$ to $-25^{\circ}$ longitude bin is coincident with the overall peak in number counts seen in Fig. 9, and the dense ridge of sources identified in the longitude-velocity domain (Figs 4 and 13) is discussed in Green et al. (2011). Given the approximately proportional distribution between bright and faint sources elsewhere in the plane (and the luminosity function described in the previous sction), the higher number of sources alone should not account for a larger proportion of brighter sources. This longitude bin corresponds to a combination of the following: the close proximity of the CarinaSagittarius and Crux-Scutum arms (within a few kpc of us); the tangent of the 3-kpc arms/ring; and the origin of the Perseus arm.

As the distribution in Fig. 22 is divided on peak flux density and not luminosity, the close proximity of the Carina-Sagittarius and Crux-Scutum arms could contribute to a larger abundance of apparently brighter sources, but the fact that it is also present in the luminosity in Fig. 23 negates this. The tangent of the 3-kpc arms/ring is only likely to contribute more sources to the sample, rather than brighter sources (unless the conditions of the 3-kpc arms/ring are special) and the Perseus arm origin could induce brighter masers only if it uniquely affected the maser physics. Brighter sources can be produced by greater pumping through higher infrared, and thus dust, temperatures, longer coherence length or higher methanol abundance (Cragg, Sobolev \& Godfrey 2002, 2005). Variations of the first of these, the dust temperature, are speculated to induce the periodic flaring seen in some maser sources (Section 4.5), and so we could, in turn, postulate that the dynamics of the arm origin interacting with the 3-kpc arms/ring are causing a systematic rise in dust temperature, which is then producing brighter masers. A preliminary comparison for this longitude bin with the results of the ATLASGAL survey (Guzmán et al. 2015) indicates that the masers associated with the arm origin (broadly those with velocities more negative than $-75 \mathrm{~km} \mathrm{~s}^{-1}$ ) have marginally higher mid-infrared temperatures than those not associated (although the limited number of sources restricts the statistical significance). You would also expect to see overabundances at the other arm origins in Figs 22 and 23 , and on closer inspection, there are indications of higher 


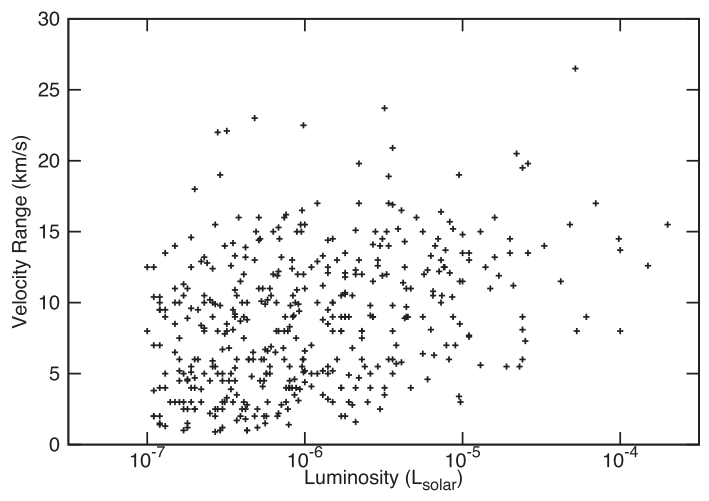

Figure 24. Luminosity compared with velocity range for sources with peak flux densities greater than $10 \sigma$ and luminosities greater than $10^{-7} \mathrm{~L}_{\odot}$.

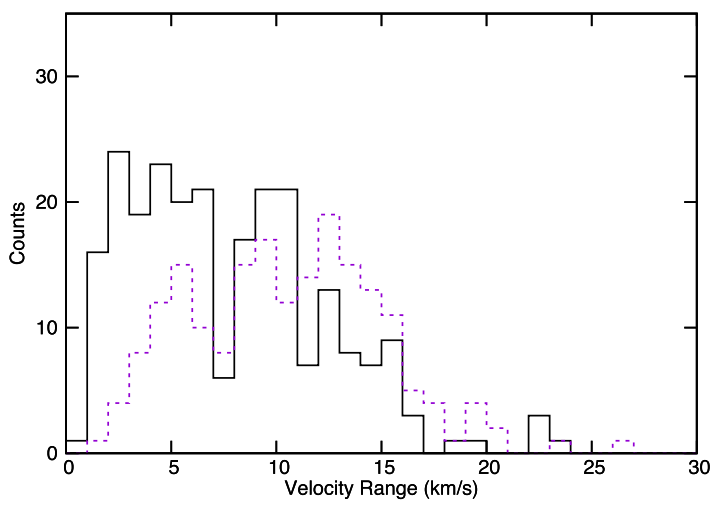

Figure 25. Source counts for given velocity ranges, split by luminosity, with those less than $10^{-6} \mathrm{~L}_{\odot}$ in solid black lines and those greater than $10^{-6} \mathrm{~L}_{\odot}$ in dashed purple lines. The luminosities are based only on sources with peak flux densities greater than $10 \sigma$ and luminosities greater than $10^{-7} \mathrm{~L} \odot$.

proportions of brighter masers at approximately $-10^{\circ}$ (Perseus), $+10^{\circ}$ (Norma) and $+20^{\circ}$ (Crux-Scutum).

\subsubsection{Distribution of velocity widths with luminosity}

The variation of velocity range (as defined in Section 4.4) with luminosity is shown in Fig. 24, and the distribution of velocity ranges above and below a luminosity threshold of $10^{-6} \mathrm{~L}_{\odot}$ is shown in Fig. 25. For both figures, only sources with peak flux densities exceeding $10 \sigma$ are included (to ensure that we are not biased by sensitivity) and those exceeding luminosities of $10^{-7} \mathrm{~L} \odot$ (to avoid distance/luminosity bias). Fig. 24 shows an approximate trend for higher luminosity sources to have larger velocity ranges; however, Fig. 25 highlights that there is a deficit of high-luminosity sources with small velocity ranges (the distribution is rising above $2.5 \mathrm{~km} \mathrm{~s}^{-1}$ and approximately flat to $15 \mathrm{~km} \mathrm{~s}^{-1}$, compared with an approximately linear increase with decreasing velocity range for the lower luminosity sample). The $<10^{-6} \mathrm{~L}_{\odot}$ sample has a mean velocity range of $7.6 \pm 0.3 \mathrm{~km} \mathrm{~s}^{-1}$, and the $>10^{-6} \mathrm{~L}_{\odot}$ sample has a mean velocity range of $10.3 \pm 0.3 \mathrm{~km} \mathrm{~s}^{-1}$, with a two-sample Kolomogorov-Smirnov test showing that the probability of the two groups being from the same distribution is $1.02 \times 10^{-6}$. Similarly, if we limit the samples to those within a distance range of $2-5 \mathrm{kpc}$, we find that the $<10^{-6} \mathrm{~L}_{\odot}$ sample is $8.5 \pm 0.5 \mathrm{~km} \mathrm{~s}^{-1}$ and the $>10^{-6} \mathrm{~L}_{\odot}$ sample is $12.2 \pm 0.7 \mathrm{~km} \mathrm{~s}^{-1}$, with a two-sample Kolomogorov-Smirnov test showing that the probability of the two groups being from the same distribution is $2.00 \times$ $10^{-4}$. The implication is that physically more luminous masers have a broader variation in the range of velocities over which emission is seen, in turn implying that a broader region of the host object is masing. This could be the maser emission reflecting the velocity dispersion of several protostars within the parent molecular cloud as a function of mass.

As noted, the maser luminosity is a product of the methanol abundance, infrared pumping and the velocity coherence. If the increased velocity range was due to the methanol abundance, it would require increased abundance throughout the source object to produce a wider velocity range. If it was due to the infrared pumping, it would require a brighter infrared flux that reaches further through the source object. Finally, if it was due to the velocity coherence, it would require more of the regions within the source having longer path lengths of coherent velocities. The last of these seems physically unlikely, and the methanol abundance and infrared flux, however, could be a result of the evolution of the source object: the increased UV of the evolving star(s) releasing more methanol to the gas phase and heating more of the dust within the object (see also Breen et al. 2011), evidenced in a possible correlation between maser luminosity and bolometric luminosity (Urquhart et al. 2013).

\section{SUMMARY}

With the completion of the MMB Survey catalogue, we present the statistics and Galactic properties of the largest sample of $6668-\mathrm{MHz}$ methanol masers. The 972 detections have a peak flux density distribution tending towards a peak at the survey sensitivity and an integrated flux density distribution exhibiting a broken power law with a break at $10 \mathrm{Jy} \mathrm{km} \mathrm{s}^{-1}$. The longitude distribution peaks at $20^{\circ}<|b|<30^{\circ}$, and the latitude distribution has an FWHM of 0.54 . There is a tendency for narrower velocity widths for weaker sources. Source variability across the survey suggests that weaker masers ( $\leq 1.5 \mathrm{Jy}$ ) have a tendency to exhibit higher variability $(\sim 35$ per cent mean variation). The MMB sources broadly trace the dense $\mathrm{CO}$ and $\mathrm{H}$ I emission, highlighting, on one hand, that the masers do not exhibit large anomalous velocities, and, on the other hand, confirming the dense $\mathrm{H}_{\mathrm{I}}$ and $\mathrm{CO}$ trace spiral arms.

We determine distances for the masers, adding H ISA allocations to those published previously, together with also utilizing a recent parallax-based Bayesian probability approach for comparison. For the resultant properties, we find broadly comparable results between the distance methods, with an expected tendency for more refined structure in the first Galactic quadrant for the Bayesian approach (where a significant number of maser parallax measurements contribute). We find scaleheights for the distributions to be comparable to other tracers, but with the Bayesian approach producing a larger value. The masers are seen to be an effective tracer of the structure of our Galaxy, the spiral arms, and the inner Galaxy structures of the 3-kpc arms/ring and the long Galactic bar.

We present the luminosity distribution for the methanol maser population, and present a full distribution fit (lognormal) and a power-law fit to the luminosities above the nominal peak of the distribution. We see brighter masers (both in flux density and luminosity) towards the spiral arm origins, and we see evidence for a correlation between increased luminosity and increased velocity width of masers, potentially a result of more luminous masers tracing more evolved host objects. 


\section{ACKNOWLEDGEMENTS}

With heartfelt sincerity this paper is dedicated to the legendary Jims, R. J. Cohen and J. L. Caswell, whose influence on this project, and the careers of several of the authors, has been profound.

We thank the anonymous referee for their comments, which enhanced and clarified this paper. The Parkes Observatory and the ATCA are part of the Australia Telescope, which is funded by the Commonwealth of Australia for operation as a National Facility managed by CSIRO. The authors greatly thank the engineering staff both at the Australia Telescope National Facility and at the Jodrell Bank Observatory, and the staff at the Parkes observatory, for ensuring the smooth running of the observations. The authors thank Joel Stedman for his efforts in examining $\mathrm{H}_{\mathrm{I}}$ spectra as part of an SKA work experience programme. For this work, SLB was supported by an Australian Research Council DECRA Fellowship (project number DE130101270) and a L'Oréal UNESCO for Women in Science Fellowship. NMM-G is the recipient of an Australian Research Council Future Fellowship.

\section{REFERENCES}

Anderson L. D., Bania T. M., 2009, ApJ, 690, 706

Anderson L. D., Bania T. M., Balser D. S., Rood R. T., 2011, ApJS, 194, 32 Anderson L. D., Bania T. M., Balser D. S., Rood R. T., 2012, ApJ, 754, 62 Araya E., Hofner P., Churchwell E., Kurtz S., 2002, ApJS, 138, 63

Araya E. D., Hofner P., Goss W. M., Kurtz S., Richards A. M. S., Linz H., Olmi L., Sewiło M., 2010, ApJ, 717, L133

Avison A. et al., 2016, MNRAS, 461, 136

Balser D. S., Rood R. T., Bania T. M., Anderson L. D., 2011, ApJ, 738, 27

Benjamin R. A. et al., 2005, ApJ, 630, L149

Breen S. L., Ellingsen S. P., Caswell J. L., Green J. A., Fuller G. A., Voronkov M. A., Quinn L. J., Avison A., 2011, ApJ, 733, 80

Breen S. L., Ellingsen S. P., Caswell J. L., Green J. A., Voronkov M. A., Fuller G. A., Quinn L. J., Avison A., 2012, MNRAS, 426, 2189

Breen S. L., Ellingsen S. P., Contreras Y., Green J. A., Caswell J. L., Stevens J. B., Dawson J. R., Voronkov M. A., 2013, MNRAS, 435, 524

Breen S. L. et al., 2015, MNRAS, 450, 4109

Breen S. L., Ellingsen S. P., Caswell J. L., Green J. A., Voronkov M. A., Avison A., Fuller G. A., Quinn L. J., 2016, MNRAS, 459, 4066

Brunthaler A. et al., 2011, Astron. Nachr., 332, 461

Burton W. B., Liszt H. S., Baker P. L., 1978, ApJ, 219, L67

Cabrera-Lavers A., González-Fernández C., Garzón F., Hammersley P. L., López-Corredoira M., 2008, A\&A, 491, 781

Caswell J. L., 1996a, MNRAS, 279, 79

Caswell J. L., 1996b, in Gredel R., ed., ASP Conf. Ser. Vol. 102, Masers and Star Formation Near the Galactic Centre. Astron. Soc. Pac., San Francisco, p. 247

Caswell J. L., Haynes R. F., 1983, Aust. J. Phys., 36, 361

Caswell J. L., Murray J. D., Roger R. S., Cole D. J., Cooke D. J., 1975, A\&A, 45, 239

Caswell J. L., Vaile R. A., Ellingsen S. P., Whiteoak J. B., Norris R. P., 1995, MNRAS, 272, 96

Caswell J. L., Vaile R. A., Ellingsen S. P., 1995, Publ. Astron. Soc. Aust., 12,37

Caswell J. L. et al., 2010, MNRAS, 404, 1029

Caswell J. L. et al., 2011, MNRAS, 417, 1964

Cheng J. Y. et al., 2012, ApJ, 746, 149

Contreras Y. et al., 2013, A\&A, 549, A45

Cragg D. M., Sobolev A. M., Godfrey P. D., 2002, MNRAS, 331, 521

Cragg D. M., Sobolev A. M., Godfrey P. D., 2005, MNRAS, 360, 533

Cyganowski C. J., Brogan C. L., Hunter T. R., Churchwell E., 2009, ApJ, 702,1615

Dame T. M., Hartmann D., Thaddeus P., 2001, ApJ, 547, 792

Elitzur M. ed. 1992, Astrophysics and Space Science Library, Vol. 170, Astronomical Masers. Kluwer, Dordrecht
Ellingsen S. P., 2007, MNRAS, 377, 571

Ellingsen S. P., von Bibra M. L., McCulloch P. M., Norris R. P., Deshpande A. A., Phillips C. J., 1996, MNRAS, 280, 378

Fish V. L., Reid M. J., Wilner D. J., Churchwell E., 2003, ApJ, 587, 701

Fujisawa K. et al., 2014a, PASJ, 66. 78

Fujisawa K. et al., 2014b, PASJ, 66, 109

Goedhart S., Gaylard M. J., van der Walt D. J., 2003, MNRAS, 339, L33

Goedhart S., Gaylard M. J., van der Walt D. J., 2004, MNRAS, 355, 553

Goedhart S., Langa M. C., Gaylard M. J., van der Walt D. J., 2009, MNRAS, 398, 995

Goedhart S., Maswanganye J. P., Gaylard M. J., van der Walt D. J., 2014, MNRAS, 437, 1808

Green J. A., McClure-Griffiths N. M., 2011, MNRAS, 417, 2500

Green J. A. et al., 2008, MNRAS, 385, 948

Green J. A. et al., 2009a, MNRAS, 392, 783

Green J. A., McClure-Griffiths N. M., Caswell J. L., Ellingsen S. P., Fuller G. A., Quinn L., Voronkov M. A., 2009b, ApJ, 696, L156

Green J. A. et al., 2010, MNRAS, 409, 913

Green J. A. et al., 2011, ApJ, 733, 27

Green J. A. et al., 2012, MNRAS, 420, 3108

Guzmán A. E., Sanhueza P., Contreras Y., Smith H. A., Jackson J. M., Hoq S., Rathborne J. M., 2015, ApJ, 815, 130

Hammersley P. L., Garzón F., Mahoney T. J., López-Corredoira M., Torres M. A. P., 2000, MNRAS, 317, L45

Heeschen D. S., 1955, ApJ, 121, 569

Kawamura A., Onishi T., Yonekura Y., Dobashi K., Mizuno A., Ogawa H., Fukui Y., 1998, ApJS, 117, 387

Kolpak M. A., Jackson J. M., Bania T. M., Clemens D. P., Dickey J. M., 2003, ApJ, 582, 756

Krishnan V. et al., 2015, ApJ, 805, 129

Krishnan V., Ellingsen S. P., Reid M. J., Bignall H. E., McCallum J., Phillips C. J., Reynolds C., Stevens J., 2017, MNRAS, 465, 1095

Kuchar T. A., Bania T. M., 1994, ApJ, 436, 117

Kurayama T., Nakagawa A., Sawada-Satoh S., Sato K., Honma M., Sunada K., Hirota T., Imai H., 2011, PASJ, 63, 513

Liszt H. S., Burton W. B., Bania T. M., 1981, ApJ, 246, 74

Lumsden S. L., Hoare M. G., Urquhart J. S., Oudmaijer R. D., Davies B., Mottram J. C., Cooper H. D. B., Moore T. J. T., 2013, ApJS, 208, 11

McClure-Griffiths N. M., Dickey J. M., Gaensler B. M., Green A. J., Haverkorn M., Strasser S., 2005, ApJs, 158, 178

McClure-Griffiths N. M. et al., 2009, ApJS, 181, 398

McMillan P. J., Binney J. J., 2010, MNRAS, 402, 934

Malyshev A. V., Sobolev A. M., 2003, Astron. Astrophys. Trans., 22, 1

Maswanganye J. P., Gaylard M. J., Goedhart S., Walt D. J. v. d., Booth R. S., 2015, MNRAS, 446, 2730

Maswanganye J. P., van der Walt D. J., Goedhart S., Gaylard M. J., 2016, MNRAS, 456, 4335

Menten K. M., Reid M. J., Forbrich J., Brunthaler A., 2007, A\&A, 474, 515

Minier V., Ellingsen S. P., Norris R. P., Booth R. S., 2003, A\&A, 403, 1095

Niinuma K. et al., 2011, PASJ, 63, 9

Olmi L., Araya E. D., Hofner P., Morales Ortiz J., Moscadelli L., Pestalozzi M., 2014, A\&A, 566, 16

Pandian J. D., Goldsmith P. F., Deshpande A. A., 2007, ApJ, 656, 255

Pestalozzi M. R., Minier V., Booth R. S., 2005, A\&A, 432, 737

Pestalozzi M. R., Chrysostomou A., Collett J. L., Minier V., Conway J., Booth R. S., 2007, A\&A, 463, 1009

Phillips C. J., Norris R. P., Ellingsen S. P., Rayner D. P., 1998, MNRAS, 294, 265

Reid M. J. et al., 2009, ApJ, 700, 137

Reid M. J., Menten K. M., Brunthaler A., Zheng X. W., Moscadelli L., Xu Y., 2009, ApJ, 693, 397

Reid M. J. et al., 2014, ApJ, 783, 130

Reid M. J., Dame T. M., Menten K. M., Brunthaler A., 2016, ApJ, 823, 77

Roman-Duval J., Jackson J. M., Heyer M., Johnson A., Rathborne J., Shah R., Simon R., 2009, ApJ, 699, 1153

Russeil D. et al., 2011, A\&A, 526, A151

Rygl K. L. J., Brunthaler A., Reid M. J., Menten K. M., van Langevelde H. J., Xu Y., 2010, A\&A, 511, A2 
Sato M. et al., 2014, ApJ, 793, 72

Schönrich R., Binney J., Dehnen W., 2010, MNRAS, 403, 1829

Sjouwerman L. O., Murray C. E., Pihlström Y. M., Fish V. L., Araya E. D., 2010, ApJL, 724, L158

Stil J. M. et al., 2006, AJ, 132, 1158

Svoboda B. E. et al., 2016, ApJ, 822, 59

Szymczak M., Wolak P., Bartkiewicz A., van Langevelde H. J., 2011, A\&A, 531, L3

Szymczak M., Wolak P., Bartkiewicz A., 2015, MNRAS, 448, 2284

Taylor J. H., Cordes J. M., 1993, ApJ, 411, 674

Urquhart J. S. et al., 2011, MNRAS, 410, 1237

Urquhart J. S. et al., 2013, MNRAS, 431, 1752

Urquhart J. S., Figura C. C., Moore T. J. T., Hoare M. G., Lumsden S. L., Mottram J. C., Thompson M. A., Oudmaijer R. D., 2014a, MNRAS, 437, 1791

Urquhart J. S. et al., 2014b, MNRAS, 443, 1555

Urquhart J. S. et al., 2015, MNRAS, 446, 3461

Vallée J. P., 2016, ApJ, 821, 53

van der Walt J., 2005, MNRAS, 360, 153

Voronkov M. A., Caswell J. L., Ellingsen S. P., Green J. A., Breen S. L., 2014, MNRAS, 439, 2584

Wu Y. W. et al., 2014, A\&A, 566, A17

Xu Y., Zheng X.-W., Jiang D.-R., 2003, Chin. J. Astron. Astrophys., 3, 49

Xu Y., Li J. J., Hachisuka K., Pandian J. D., Menten K. M., Henkel C., 2008, A\&A, 485, 729

Zhang B., Zheng X. W., Reid M. J., Menten K. M., Xu Y., Moscadelli L., Brunthaler A., 2009, ApJ, 693, 419

Zhang B., Reid M. J., Menten K. M., Zheng X. W., Brunthaler A., Dame T. M., Xu Y., 2013, ApJ, 775, 79

Zhang B. et al., 2014, ApJ, 781, 89

\section{SUPPORTING INFORMATION}

Supplementary data are available at MNRAS online.

Appendix A. Kinematic distances.

Appendix B. Parallax-based distances.

Please note: Oxford University Press is not responsible for the content or functionality of any supporting materials supplied by the authors. Any queries (other than missing material) should be directed to the corresponding author for the article.
${ }^{1}$ CSIRO Astronomy and Space Science, Australia Telescope National Facility, PO Box 76, Epping, NSW 1710, Australia

${ }^{2}$ SKA Organisation, Jodrell Bank Observatory, Lower Withington, Macclesfield SK11 9DL, UK

${ }^{3}$ Sydney Institute for Astronomy (SiFA), School of Physics, University of Sydney, NSW 2006, Australia

${ }^{4}$ Jodrell Bank Centre for Astrophysics, Alan Turing Building, School of Physics and Astronomy, The University of Manchester, Manchester M13 9PL, UK

${ }^{5}$ UK ALMA Regional Centre Node, Alan Turing Building, School of Physics and Astronomy, The University of Manchester, Manchester M13 9PL, UK

${ }^{6}$ Research School of Astronomy and Astrophysics, The Australian National University, Canberra, ACT 2611, Australia

${ }^{7}$ School of Physical Sciences, University of Tasmania, Private Bag 37, Hobart, TAS 7001, Australia

${ }^{8}$ Astro Space Centre, Profsouznaya st. 84/32, 117997 Moscow, Russia

${ }^{9}$ Murdoch University, 90 South Street, Murdoch, WA 6150, Australia

${ }^{10}$ School of Physics, University of New South Wales, Sydney, NSW 2052, Australia

${ }^{11}$ Armagh Observatory and Planetarium, College Hill, Armagh BT61 9DG, $U K$

${ }^{12}$ Centre for Astrophysics Research, Science and Technology Research Institute, University of Hertfordshire, College Lane, Hatfield AL10 9AB, UK

${ }^{13}$ Department of Physics and Astronomy, Cardiff University, 5 The Parade, Cardiff CF24 3YB, UK

${ }^{14}$ School of Physics and Astronomy, University of Leeds, Leeds LS2 9JT, UK

${ }^{15}$ Astrophysics Group, Department of Physics, Bristol University, Tyndall Avenue, Bristol BS8 1TL, UK

${ }^{16}$ Istituto di Astrofisica e Planetologia Spaziali IAPS - INAF, via del Fosso del cavaliere 100, I-00133 Roma, Italy

${ }^{17}$ Crompton House Church of England School, Rochdale Road, Shaw, Greater Manchester, Oldham OL2 7HS, UK

${ }^{18}$ International Centre for Radio Astronomy Research, Curtin University, Building 610, 1 Turner Avenue, Bentley, WA 6102, Australia

${ }^{19}$ Jeremiah Horrocks Institute for Mathematics, Physics and Astronomy, University of Central Lancashire, PR1 2HE, UK

${ }^{20}$ Department of Physics and Astronomy, University College London, Gower Street, London WC1E 6BT, UK

This paper has been typeset from a $\mathrm{T}_{\mathrm{E}} \mathrm{X} / \mathrm{L} \mathrm{T} \mathrm{E} \mathrm{X}$ file prepared by the author. 